\title{
Drinfeld realization of the elliptic Hall algebra
}

\author{
Olivier Schiffmann
}

Received: 15 April 2010 / Accepted: 17 June 2011 / Published online: 9 July 2011

(C) Springer Science+Business Media, LLC 2011

\begin{abstract}
We give a new presentation of the Drinfeld double $\mathcal{E}$ of the (spherical) elliptic Hall algebra $\mathcal{E}^{+}$introduced in our previous work (Burban and Schiffmann in Duke Math. J. preprint math.AG/0505148, 2005). This presentation is similar in spirit to Drinfeld's 'new realization' of quantum affine algebras. This answers, in the case of elliptic curves, a question of Kapranov concerning functional relations satisfied by (principal, unramified) Eisenstein series for $G L(n)$ over a function field. It also provides proofs of some recent conjectures of Feigin, Feigin, Jimbo, Miwa and Mukhin (arXiv:1002.3100, 2010).
\end{abstract}

Keywords Hall algebras · Cherednik algebras · Shuffle algebras · Drinfeld new realization

\section{Introduction}

In the seminal paper [11], M. Kapranov initiated the study of the so-called Hall algebra $\mathbf{H}_{X}$ of the category of coherent sheaves over a smooth projective curve $X$ (defined over a finite field). It may be interpreted in the language of automorphic forms as an algebra of (unramified) automorphic forms for the collection of groups $G L(n)(n \geq 1)$ over the function field of $X$, with the product being given by the parabolic induction $G L(n) \times G L(m) \rightarrow G L(n+m)$. On the other hand, the well-known analogy between Hall algebras of abelian categories and quantum groups (discovered by Ringel [12]) suggests that $\mathbf{H}_{X}$ should behave like the positive half of some 'quantum enveloping algebra'. Indeed, as observed by Kapranov, the functional equations for Eisenstein series (which express the invariance of these Eisenstein series under the action of the

O. Schiffmann $(\bowtie)$

Département de Mathématiques, Faculté des Sciences d’Orsay, Université Paris Sud, 91405 Orsay

Cedex, France

e-mail: olivier.schiffmann@math.u-psud.fr 
Weyl group, and which are quadratic relations) resemble very much the relations appearing in Drinfeld's 'new realization' of quantum affine algebras (see [3]). In fact, they coincide precisely with these relations when $X=\mathbb{P}^{1}$ (see $[1,11]$ ). As soon as $X$ is of genus at least one, however, the functional equations do not suffice to determine the Hall algebra $\mathbf{H}_{X}$, and one has to look for some new, higher rank 'functional equations' satisfied by Eisenstein series.

The aim of this paper is to determine these higher rank functional equations when $X$ is an elliptic curve. More precisely, we consider the Drinfeld double $\mathbf{U}_{X}$ of the spherical Hall algebra $\mathbf{U}_{X}^{+}$of $X$, i.e. the (Drinfeld double of the) subalgebra of $\mathbf{H}_{X}$ generated by the characteristic functions of the connected components $\operatorname{Pic}^{d}(X)$ of the Picard group together with the characteristic functions of the stacks of torsion sheaves on $X$. We show that $\mathbf{U}_{X}$ can be abstractly presented as an algebra generated by the above elements subject to the standard (quadratic) functional equations plus one set of cubic relations (see Theorem 4, equations (5.5)). Our method is rather brutal and very specific to elliptic curves; it is based on a combinatorial realization of $\mathbf{U}_{X}$ given in our previous work [2] in terms of lattice paths in $\mathbb{Z}^{2}$. We nevertheless expect that our result can be extended to the whole Hall algebra $\mathbf{H}_{X}$ (of an elliptic curve) and its Drinfeld double $\mathbf{D H}_{X}{ }^{1}$

As it turns out, the (spherical) elliptic Hall algebra $\mathbf{U}_{X}$, or rather its rational form $\mathcal{E}$ appears in several other guises: as shown in [14] it projects to the spherical Cherednik algebra $\mathbf{S} \ddot{\mathbf{H}}_{n}$ for any $n \in \mathbb{Z}$ and as such may be thought of as the stable limit $\mathbf{S} \ddot{\mathbf{H}}_{\infty}$ of these Cherednik algebras; as shown in [15], $\mathcal{E}$ may be identified with a convolution algebra acting on the (equivariant) K-theory of Hilbert schemes of points in $\mathbb{C}^{2}$ and its positive half $\mathcal{E}^{>}$admits a realization as a shuffle algebra of Feigin-Odesskii type. Finally, the algebra $\mathcal{E}$ (and the presentation given in Theorem 4) also appears in recent work of Feigin, Feigin, Jimbo, Miwa and Mukhin (see [5, 6, 9]) where its representation theory is studied. It also appears in [7, 8]. Our results verify some of the conjectures presented in loc. cit. (see Sect. 4).

The plan of the paper is the following: after introducing the algebra $\mathbf{U}_{X}$, its rational form $\mathcal{E}$ and after recalling their relation to DAHAs and shuffle algebras (Sects. 2-4) we state our main theorem giving a Drinfeld realization of $\mathcal{E}$ in Sect. 5 including its Hopf algebra structure. Section 6 is dedicated to the (elementary but intricate) proof of that result.

\section{Recollections on the elliptic Hall algebra}

\section{1}

We begin with some recollections concerning the spherical Hall algebra $\mathbf{U}_{X}^{+}$of an elliptic curve $X$ defined over a finite field $\mathbb{F}_{q}$ and its Drinfeld double $\mathbf{U}_{X}$. We refer to [2] for details. Let $\operatorname{Coh}(X)$ be the category of coherent sheaves over $X$, and let $\mathcal{I}$ be the set of isomorphism classes of objects in $\operatorname{Coh}(X)$. There is a partition $\mathcal{I}=\sqcup_{r, d} \mathcal{I}_{r, d}$

\footnotetext{
${ }^{1}$ This has recently been carried out by Dragos Fratila, see [10].
} 
according to the rank and the degree of a coherent sheaf. The Euler form on the Grothendieck group $K_{0}(\operatorname{Coh}(X))$ is given by

$$
\langle\mathcal{F}, \mathcal{G}\rangle=\operatorname{rk}(\mathcal{F}) \operatorname{deg}(\mathcal{G})-r k(\mathcal{G}) \operatorname{deg}(\mathcal{F}) .
$$

Set

$$
\mathbf{H}_{X}[r, d]=\left\{f: \mathcal{I}_{r, d} \rightarrow \mathbb{C} \mid \#(\operatorname{supp} f)<\right\}=\bigoplus_{\mathcal{F} \in \mathcal{I}_{r, d}} \mathbb{C} 1_{\mathcal{F}},
$$

and put $\mathbf{H}_{X}=\bigoplus_{r, d} \mathbf{H}_{\mathbf{x}}[r, d]$, where the sums runs over all possible pairs $(r, d)$, i.e. $(r, d) \in\left(\mathbb{Z}^{2}\right)^{+} \cup\{(0,0)\}$, where

$$
\left(\mathbb{Z}^{2}\right)^{+}=\{(r, d) \mid r>0 \text { or } r=0, d>0\} .
$$

To a triple $(\mathcal{F}, \mathcal{G}, \mathcal{H})$ of coherent sheaves we associate the finite set

$$
\mathcal{P}_{\mathcal{F}, \mathcal{G}}^{\mathcal{H}}=\{(\phi, \psi) \mid 0 \rightarrow \mathcal{G} \stackrel{\phi}{\rightarrow} \mathcal{H} \stackrel{\psi}{\rightarrow} \mathcal{F} \rightarrow 0 \text { is exact }\}
$$

and we set $P_{\mathcal{F}, \mathcal{G}}^{\mathcal{H}}=\# \mathcal{P}_{\mathcal{F}, \mathcal{G}}^{\mathcal{H}}$. We write $a_{\mathcal{K}}=\# \operatorname{Aut}(\mathcal{K})$ for a coherent sheaf $\mathcal{K}$. We set $v=q^{-1 / 2}$. Define an associative product on $\mathbf{H}_{X}$ by the formula

$$
1_{\mathcal{F}} \cdot 1_{\mathcal{G}}=v^{-\langle\mathcal{F}, \mathcal{G}\rangle} \sum_{\mathcal{H}} \frac{1}{a_{\mathcal{F}} a_{\mathcal{G}}} P_{\mathcal{F}, \mathcal{G}}^{\mathcal{H}} 1_{\mathcal{H}},
$$

and a coassociative coproduct by

$$
\Delta\left(1_{\mathcal{H}}\right)=\sum_{\mathcal{F}, \mathcal{G}} v^{-\langle\mathcal{F}, \mathcal{G}\rangle} \frac{P_{\mathcal{F}, \mathcal{G}}^{\mathcal{H}}}{a_{\mathcal{H}}} 1_{\mathcal{F}} \otimes 1_{\mathcal{G}}
$$

There is a natural pairing on $\mathbf{H}_{X}$ given by

$$
\left(1_{\mathcal{F}}, 1_{\mathcal{G}}\right)=\delta_{\mathcal{F}, \mathcal{G}} \frac{1}{a_{\mathcal{F}}} .
$$

Then $\left(\mathbf{H}_{X}, \cdot, \Delta\right)$ is a (topological) bialgebra (see e.g. [13]). Moreover, the pairing ( , ) is a Hopf pairing and it is nondegenerate, making $\mathbf{H}_{X}$ a self-dual bialgebra.

For $d \in \mathbb{Z}$ let $1_{\text {Pic }^{d}(X)}=\sum_{\mathcal{L} \in \text { Pic }^{d}(X)} 1_{\mathcal{L}}$ be the characteristic function of the Picard variety of degree $d$; for $l \geq 1$ let $1_{T o r^{l}(X)}=\sum_{\mathcal{T} \in \operatorname{Tor}^{l}(X)} 1_{\mathcal{T}}$ be the characteristic function of the set of torsion sheaves of degree $l$; more generally, for any $(r, d) \in\left(\mathbb{Z}^{2}\right)^{+}$ let $1_{r, d}^{s s}$ be the characteristic function of the set of semistable sheaves of rank $r$ and degree $d$. By definition, the spherical Hall algebra $\mathbf{U}_{X}^{+}$of $X$ is the subalgebra of $\mathbf{H}_{X}$ generated by $\left\{1_{\operatorname{Pic}^{d}(X)} \mid d \in \mathbb{Z}\right\} \cup\left\{1_{\operatorname{Tor}^{l}(X)} \mid l \in \mathbb{N}\right\}$. One shows that it is a sub bialgebra of $\mathbf{H}_{X}$, and that it contains the element $1_{r, d}^{s s}$ for any $r, d$.

Finally, let $\mathbf{U}_{X}$ be the Drinfeld double of $\mathbf{U}_{X}^{+}$; it is a an algebra generated by two copies $\mathbf{U}_{X}^{+}$and $\mathbf{U}_{X}^{-}$of the spherical Hall algebra $\mathbf{U}_{X}^{+}$(see e.g. [2, Sect. 3] for more details). If $u$ belongs to the spherical Hall algebra, we denote by $u^{+}$and $u^{-}$the corresponding elements in $\mathbf{U}_{X}^{+}$and $\mathbf{U}_{X}^{-}$. 
We refer to [11] for the interpretation of $\mathbf{H}_{X}$ or $\mathbf{U}_{X}$ in the language of automorphic forms over function fields.

The spherical Hall algebras $\mathbf{U}_{X}^{+}$and $\mathbf{U}_{X}$ admit the following combinatorial presentation, obtained in [2]. Let $\sigma, \bar{\sigma}$ be the two Weil numbers of $X$, i.e. the Frobenius eigenvalues in $H^{1}\left(\bar{X}, \overline{\mathbb{Q}_{1}}\right)$. Thus we have $\sigma \bar{\sigma}=q=v^{-2}$. For $i \geq 1$ we put

$$
\alpha_{i}=\alpha_{i}(\sigma, \bar{\sigma})=\left(1-\sigma^{i}\right)\left(1-\bar{\sigma}^{i}\right)\left(1-(\sigma \bar{\sigma})^{-i}\right) / i
$$

Let us set $\left(\mathbb{Z}^{2}\right)^{*}=\mathbb{Z}^{2} \backslash\{(0,0)\}$,

$$
\begin{gathered}
\left(\mathbb{Z}^{2}\right)^{+}=\left\{(p, q) \in \mathbb{Z}^{2} \mid p>0 \text { or } p=0, q>0\right\}, \\
\left(\mathbb{Z}^{2}\right)^{>}=\left\{(p, q) \in \mathbb{Z}^{2} \mid p>0\right\},
\end{gathered}
$$

and $\left(\mathbb{Z}^{2}\right)^{-}=-\left(\mathbb{Z}^{2}\right)^{+},\left(\mathbb{Z}^{2}\right)^{<}=-\left(\mathbb{Z}^{2}\right)^{>}$. For any $\mathbf{x}=(p, q) \in\left(\mathbb{Z}^{2}\right)^{*}$ we put $\partial(x)=$ g.c.d. $(p, q) \in \mathbb{N}$ and call this positive number the length of $\mathbf{x}$. We will say that $\mathbf{x}$ is primitive if $\partial(\mathbf{x})=1$. Finally, for a pair of non-collinear $(\mathbf{x}, \mathbf{y}) \in\left(\mathbb{Z}^{2}\right)^{*}$ we set $\epsilon_{\mathbf{x}, \mathbf{y}}=$ $\operatorname{sign}(\operatorname{det}(\mathbf{x}, \mathbf{y})) \in\{ \pm 1\}$. We also let $\Delta_{\mathbf{x}, \mathbf{y}}$ stand for the triangle in $\mathbb{Z}^{2}$ with vertices $\{(0,0), \mathbf{x}, \mathbf{x}+\mathbf{y}\}$ (Fig. 1).

Because of the relation to Hall algebras of elliptic curves (see below), we usually refer to the first component of a lattice point $\mathbf{x}=(r, d) \in \mathbb{Z}^{2}$ as its rank and to its second component as its degree.

Definition Let $\mathcal{E}_{X}$ be the unital $\mathbb{C}$-algebra generated by elements $\left\{u_{\mathbf{x}} \mid \mathbf{x} \in\left(\mathbb{Z}^{2}\right)^{*}\right\}$ modulo the following set of relations:

(i) If $\mathbf{x}, \mathbf{x}^{\prime}$ belong to the same line in $\mathbb{Z}^{2}$ then

$$
\left[u_{\mathbf{x}}, u_{\mathbf{x}^{\prime}}\right]=0 .
$$

(ii) Assume that $\mathbf{x}, \mathbf{y} \in\left(\mathbb{Z}^{2}\right)^{*}$ are such that $\mathbf{x}$ is primitive and that $\Delta_{\mathbf{x}, \mathbf{y}}$ has no interior lattice point. Then

$$
\left[u_{\mathbf{y}}, u_{\mathbf{x}}\right]=\epsilon_{\mathbf{x}, \mathbf{y}} \frac{\theta_{\mathbf{x}+\mathbf{y}}}{\alpha_{1}}
$$

Fig. 1 The triangle $\Delta_{\mathbf{x}, \mathbf{y}}$

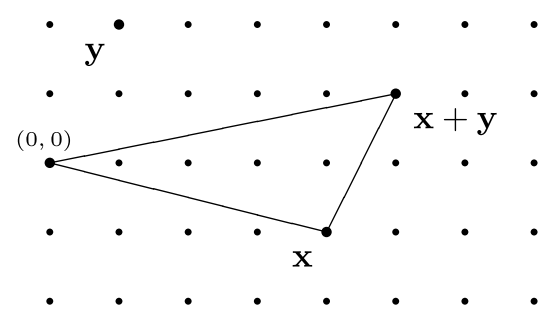


where the elements $\theta_{\mathbf{z}}, \mathbf{z} \in\left(\mathbb{Z}^{2}\right)^{*}$ are obtained by equating the Fourier coefficients of the collection of relations

$$
1+\sum_{i>0} \theta_{i \mathbf{x}_{0}} s^{i}=\exp \left(\sum_{r \geq 1} \alpha_{r} u_{r \mathbf{x}_{0}} s^{r}\right),
$$

for any primitive $\mathbf{x}_{0} \in\left(\mathbb{Z}^{2}\right)^{*}$ (observe that $\theta_{\mathbf{z}} / \alpha_{1}=u_{\mathbf{z}}$ if $\operatorname{deg}(\mathbf{z})=1$ ).

The relation with spherical Hall algebras is given in the following theorem. Set as usual $[l]=\left(v^{l}-v^{-l}\right) /\left(v-v^{-1}\right)$ and define elements $T_{r, d}$ in $\mathbf{U}_{X}^{+}$for $(r, d) \in\left(\mathbb{Z}^{2}\right)^{+}$ via the following equations

$$
1+\sum_{l \geq 1} 1_{l \mathbf{x}}^{s s}=\exp \left(\sum_{l \geq 1} \frac{1}{[l]} T_{l \mathbf{x}} s^{l}\right)
$$

for all primitive vectors $\mathbf{x}$. We also set $c_{i}=\alpha_{i} /\left(v^{-1}-v\right)$.

Theorem 1 [2] The assignment

$$
T_{1, d}^{ \pm} \mapsto c_{1} u_{ \pm 1, \pm d}, \quad T_{0, l}^{ \pm} \mapsto c_{l} u_{0, \pm l}
$$

extends to an isomorphism of algebras $\Phi: \mathbf{U}_{X} \stackrel{\sim}{\rightarrow} \mathcal{E}_{X}$. Moreover, we have $\Phi\left(T_{\mathbf{x}}^{ \pm}\right)=$ $c_{\partial(\mathbf{x})} u_{ \pm \mathbf{x}}$ for any $\mathbf{x} \in\left(\mathbb{Z}^{2}\right)^{+}$.

Observe that $\mathcal{E}_{X}$ is $\mathbb{Z}^{2}$-graded (by the rank and the degree) and carries a natural $S L(2, \mathbb{Z})$-symmetry. It corresponds to an action of $S L(2, \mathbb{Z})$ by Fourier-Mukai transforms on the derived category $D^{b}(\operatorname{Coh}(X))$. Rather than $\mathcal{E}_{X}$, we will use the rational form $\mathcal{E}_{\mathbf{K}}$ of $\mathcal{E}_{X}$ defined over the field $\mathbf{K}=\mathbb{C}(\sigma, \bar{\sigma})$, where $\sigma, \bar{\sigma}$ are now formal parameters. Since the ground field will always be $\mathbf{K}$, we will drop the index $\mathbf{K}$ from the notation.

We denote by $\mathcal{E}^{ \pm}$the subalgebra of $\mathcal{E}$ generated by $u_{\mathbf{x}}$ for $\mathbf{x} \in\left(\mathbb{Z}^{2}\right)^{ \pm}$. The following is proved in [2, Corollary 5.7]

Proposition 2.1 The algebra $\mathcal{E}^{ \pm}$is isomorphic to the algebra generated by elements $\left\{u_{\mathbf{x}}, \mathbf{x} \in\left(\mathbb{Z}^{2}\right)^{ \pm}\right\}$modulo the set of relations (2.3-2.4). Moreover there is a triangular decomposition

$$
\mathcal{E} \simeq \mathcal{E}^{+} \otimes \mathcal{E}^{-}
$$

It is also shown in [2, Corollary 6.1] that $\mathcal{E}$ is actually generated by elements $\left\{u_{r, d}\right\}$ with $-1 \leq r \leq 1$; likewise the subalgebras $\mathcal{E}^{+}$and $\mathcal{E}^{-}$are, respectively, generated by the elements $\left\{u_{1, d}, u_{0, l}\right\}$ and $\left\{u_{-1, d}, u_{0,-l}\right\}$ for $d \in \mathbb{Z}$ and $l \in \mathbb{N}$. 


\section{Link to spherical DAHAs}

\section{1}

In this section, which is included for completeness, we briefly describe the relation between the elliptic Hall algebra $\mathcal{E}$ and spherical DAHAs of type $G L(n)$. Let $\ddot{\mathbf{H}}_{n}$ be the double affine Hecke algebra of type $G L(n)$ and parameters $t=\sigma^{-1}, q=\bar{\sigma}^{-1}$. This is an algebra generated by elements $X_{i}^{ \pm 1}, Y_{i}^{ \pm 1}, T_{j}$ for $i=1, \ldots, n$ and $j=$ $1, \ldots, n-1$, subject to a set of relations which we will not write here (see [14, Sect. 2]). Let $S$ stand for the complete idempotent in the finite Hecke algebra $\mathbf{H}_{n}$ generated by $T_{1}, \ldots, T_{n-1}$ and put $\mathbf{S} \ddot{\mathbf{H}}_{n}=S \ddot{\mathbf{H}}_{n} S$. There is a well-known action of the group $S L(2, \mathbb{Z})$ on $\mathbf{S} \ddot{\mathbf{H}}_{n}$ by algebra automorphisms, and we may use it to define a unique collection of elements $P_{r, d}^{(n)}$ for $(r, d) \in \mathbb{Z}^{2} \backslash\{0\}$ satisfying $P_{0, d}^{(n)}=S \sum_{i} Y_{i}^{d} S$ if $d>0$ and

$$
P_{\gamma(r, d)}^{(n)}=\gamma \cdot P_{r, d}^{(n)}
$$

for all $\gamma \in S L(2, \mathbb{Z})$.

The following result is proved in [14, Theorem 3.1]:

Theorem 2 For any $n$ there is a surjective algebra morphism $\Psi_{n}: \mathcal{E} \rightarrow \mathbf{S} \ddot{\mathbf{H}}_{n}$ given by

$$
u_{r, d} \mapsto \frac{1}{q^{\partial(r, d)}-1} P_{r, d}^{(n)} .
$$

Moreover we have $\bigcap_{n} \operatorname{Ker} \Psi_{n}=\{0\}$.

We may think of $\mathcal{E}$ as a stable limit $\mathbf{S} \ddot{\mathbf{H}}_{\infty}$ of $\mathbf{S} \ddot{\mathbf{H}}_{n}$ as $n$ tends to infinity.

\section{Shuffle algebra presentation}

\section{1}

We now provide a shuffle realization of the positive elliptic Hall algebra $\mathcal{E}^{>}$. Such a realization was obtained in [15]. The same shuffle algebra also appears in the work of Feigin and Tsymbaliuk (see [9]).

Set

$$
\tilde{\zeta}(z)=\frac{(1-\sigma z)(1-\bar{\sigma} z)\left(1-(\sigma \bar{\sigma})^{-1} z\right)}{(1-z)}
$$

and put $\tilde{\zeta}\left(z_{1}, \ldots, z_{r}\right)=\prod_{i<j} \tilde{\zeta}\left(z_{i} / z_{j}\right)$. Following Feigin and Odesskii [4] we define an associative algebra $\mathbf{A}=\mathbf{A}_{\tilde{\zeta}(z)}$ as follows. Consider the twisted symmetrization operator

$$
\begin{aligned}
\Psi_{r}: \mathbf{K}\left[z_{1}^{ \pm 1}, \ldots, z_{r}^{ \pm 1}\right] & \rightarrow \mathbf{K}\left[z_{1}^{ \pm 1}, \ldots, z_{r}^{ \pm 1}\right]^{\mathfrak{S}_{r}} \\
P\left(z_{1}, \ldots, z_{r}\right) & \mapsto \sum_{\gamma \in \mathfrak{S}_{r}} \gamma \cdot\left(\tilde{\zeta}\left(z_{1}, \ldots, z_{r}\right) P\left(z_{1}, \ldots, z_{r}\right)\right)
\end{aligned}
$$


and set $\mathbf{A}_{r}=\operatorname{Im}\left(\Psi_{r}\right)$. Note that $\Psi_{r}$ is a $\mathbf{K}\left[z_{1}^{ \pm 1}, \ldots, z_{r}^{ \pm 1}\right]^{\mathfrak{S}_{r}}$-module morphism. There is a unique linear map $m_{r, s}: \mathbf{A}_{r} \otimes \mathbf{A}_{s} \rightarrow \mathbf{A}_{r+s}$ fitting in a commutative diagram

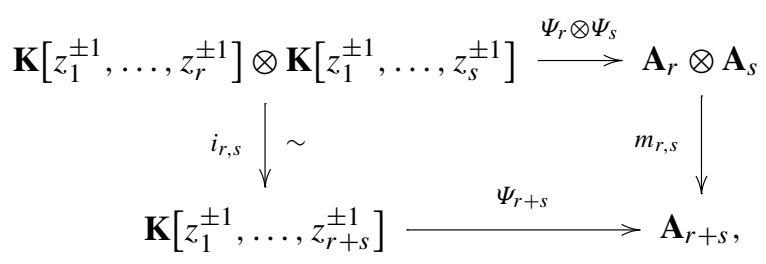

where $i_{r, s}\left(P\left(z_{1}, \ldots, z_{r}\right) \otimes Q\left(z_{1}, \ldots, z_{s}\right)\right)=P\left(z_{1}, \ldots, z_{r}\right) Q\left(z_{r+1}, \ldots, z_{r+s}\right)$. It is easy to check that the maps $m_{r, s}$ endow the space $\mathbf{A}=\mathbf{K} \oplus \bigoplus_{r>1} \mathbf{A}_{r}$ with the structure of an associative algebra. The product in $\mathbf{A}$ may be explicitly written as the shuffle operation

$h\left(z_{1}, \ldots, z_{r}\right) \cdot f\left(z_{1}, \ldots, z_{s}\right)$

$$
=\frac{1}{r ! s !} \operatorname{Sym}_{r+s}\left(\prod_{\substack{1 \leq i \leq r \\ r+1 \leq j \leq r+s}} \tilde{\zeta}\left(z_{i} / z_{j}\right) \cdot h\left(z_{1}, \ldots, z_{r}\right) f\left(z_{r+1}, \ldots, z_{r+s}\right)\right),
$$

where Sym is the usual symmetrization operator. The following is shown in [15, Theorem 10.1]:

Theorem 3 The assignment $u_{1, d} \mapsto z_{1}^{d} \in \mathbf{A}_{1}$ for $d \in \mathbb{Z}$ extends to an isomorphism of $\mathbf{K}$-algebras $\Phi: \mathcal{E}^{>} \stackrel{\sim}{\rightarrow} \mathbf{A}$.

Remark A similar shuffle realization exists for the (spherical) Hall algebra of an arbitrary smooth projective curve (see [16]).

\section{Drinfeld presentation}

This section contains our main result, i.e. a presentation of the elliptic Hall algebra $\mathcal{E}$ akin to the 'Drinfeld new realization' of quantum affine algebras.

5.1

Consider the formal series

$$
\mathbb{T}_{1}(z)=\sum_{l} u_{1, l} z^{l}, \quad \mathbb{T}_{-1}(z)=\sum_{l} u_{-1, l} z^{l},
$$

and

$$
\mathbb{T}_{0}^{+}(z)=1+\sum_{l \geq 1} \theta_{(0, l)} z^{l}, \quad \mathbb{T}_{0}^{-}(z)=1+\sum_{l \geq 1} \theta_{(0,-l)} z^{-l}
$$


We introduce the polynomials

$$
\begin{aligned}
\chi_{1}(z, w) & =(z-\sigma w)(z-\bar{\sigma} w)\left(z-(\sigma \bar{\sigma})^{-1} w\right), \\
\chi_{-1}(z, w) & =\left(z-\sigma^{-1} w\right)\left(z-\bar{\sigma}^{-1} w\right)(z-\sigma \bar{\sigma} w) .
\end{aligned}
$$

Note that $\chi_{-1}(z, w)=-\chi_{1}(w, z)$. Finally, we set as usual $\delta(x)=\sum_{l \in \mathbb{Z}} x^{l}$.

In the theorem below, the equations are to be understood formally, i.e. as equalities of Fourier coefficients of $z^{n} w^{l}$ for all $n, l \in \mathbb{Z}$. The residue operation is defined as follows: if $A(z)=\sum_{l \in \mathbb{Z}} a_{l} z^{l}$ is a formal series then $\operatorname{Res}_{z} A(z)=a_{-1}$.

Theorem 4 The elliptic Hall algebra $\mathcal{E}$ is isomorphic to the algebra generated by the Fourier coefficients of $\mathbb{T}_{-1}(z), \mathbb{T}_{1}(z), \mathbb{T}_{0}^{+}(z)$ and $\mathbb{T}_{0}^{-}(z)$, modulo the following relations, for all $\epsilon, \epsilon_{1}, \epsilon_{2} \in\{1,-1\}$ :

$$
\begin{aligned}
\mathbb{T}_{0}^{\epsilon_{1}}(z) \mathbb{T}_{0}^{\epsilon_{2}}(w) & =\mathbb{T}_{0}^{\epsilon_{2}}(w) \mathbb{T}_{0}^{\epsilon_{1}}(z), \\
\chi_{\epsilon_{1}}(z, w) \mathbb{T}_{0}^{\epsilon_{2}}(z) \mathbb{T}_{\epsilon_{1}}(w) & =\chi_{-\epsilon_{1}}(z, w) \mathbb{T}_{\epsilon_{1}}(w) \mathbb{T}_{0}^{\epsilon_{2}}(z), \\
\chi_{\epsilon}(z, w) \mathbb{T}_{\epsilon}(z) \mathbb{T}_{\epsilon}(w) & =\chi_{-\epsilon}(z, w) \mathbb{T}_{\epsilon}(w) \mathbb{T}_{\epsilon}(z), \\
{\left[\mathbb{T}_{-1}(z), \mathbb{T}_{1}(w)\right] } & =\frac{1}{\alpha_{1}}\left(\mathbb{T}_{0}^{-}(z) \delta\left(\frac{z}{w}\right)-\mathbb{T}_{0}^{+}(z) \delta\left(\frac{z}{w}\right)\right),
\end{aligned}
$$

together with the cubic relations

$$
\operatorname{Res}_{z, y, w}\left[(z y w)^{m}(z+w)\left(y^{2}-z w\right) \mathbb{T}_{\epsilon}(z) \mathbb{T}_{\epsilon}(y) \mathbb{T}_{\epsilon}(w)\right]=0,
$$

for all $m \in \mathbb{Z}$ and $\epsilon \in\{-1,1\}$.

The proof of the above theorem is given in Sect. 6. The cubic relations (5.5) may also be written more simply as follows:

$$
\left[\left[u_{1, l+1}, u_{1, l-1}\right], u_{1, l}\right]=\left[\left[u_{-1, l-1}, u_{-1, l+1}\right], u_{-1, l}\right]=0
$$

for all $l \in \mathbb{Z}$. Introduce elements $u_{0, l}$ for $l \in \mathbb{Z}^{*}$ via the formulas

$$
\log \left(1+\sum_{i>0} \theta_{(0, \pm i)} s^{i}\right)=\sum_{r \geq 1} \alpha_{r} u_{( \pm r, 0)} s^{r} .
$$

As a consequence of the identity

$$
\sum_{l \geq 1} \alpha_{l} \frac{w^{l}}{z^{l}}=\log \left(\chi_{1}(z, w)\right)-\log \left(\chi_{-1}(z, w)\right)
$$

relation (5.2) is equivalent to

$$
\left[u_{0, \pm l}, u_{1, n}\right]= \pm u_{1, n \pm l} .
$$


Note that the $S L(2, \mathbb{Z})$-symmetry of $\mathcal{E}$ is broken in the above presentation. However, there is still a natural symmetry by the unipotent subgroup $\left(\begin{array}{ll}1 & \mathbb{Z} \\ 0 & 1\end{array}\right) \subset S L(2, \mathbb{Z})$, given by

$$
\left(\begin{array}{cc}
1 & n \\
0 & 1
\end{array}\right) \cdot u_{ \pm 1, l}=u_{ \pm 1, l \pm n}, \quad\left(\begin{array}{cc}
1 & n \\
0 & 1
\end{array}\right) \cdot \theta_{0, l}=\theta_{0, l} .
$$

The following remark is noteworthy:

Lemma 5.1 The cubic relations (5.6) for all $l \in \mathbb{Z}$ are implied by (5.2) and a single cubic relation

$$
\left[\left[u_{1,1}, u_{1,-1}\right], u_{1,0}\right]=\left[\left[u_{-1,-1}, u_{-1,1}\right], u_{-1,0}\right]=0 .
$$

Proof The idea is to explicitly realize the action of the unipotent symmetry group $\left(\begin{array}{ll}1 & \mathbb{Z} \\ 0 & 1\end{array}\right)$ in terms of the adjoint action of the rank zero operators $u_{0, l}$. For this, consider the vector space map

$$
J: \mathbf{K}\left[x_{1}^{ \pm 1}, x_{2}^{ \pm 1}, x_{3}^{ \pm 1}\right] \rightarrow \mathcal{E}, \quad x_{1}^{l_{1}} x_{2}^{l_{2}} x_{3}^{l_{3}} \mapsto u_{1, l_{1}} u_{1, l_{2}} u_{1, l_{3}} .
$$

By relation (5.8) the adjoint action of an element $u_{0, l}, l \geq 1$ satisfies

$$
\operatorname{ad}\left(u_{0, l}\right) J\left(P\left(x_{1}, x_{2}, x_{3}\right)\right)=J\left(p_{l} \cdot P\left(x_{1}, x_{2}, x_{3}\right)\right),
$$

where $p_{l}=x_{1}^{l}+x_{2}^{l}+x_{3}^{l}$. Since the power sum functions $p_{l}$ generate the algebra of symmetric polynomials, there exists for any $k \geq 1$ an operator $v_{k}$ (an explicit polynomial in the adjoint operators $\operatorname{ad}\left(u_{0, l}\right)$ satisfying $v_{k} \cdot J\left(P\left(x_{1}, x_{2}, x_{3}\right)\right)=$ $\left.J\left(\left(x_{1} x_{2} x_{3}\right)^{k} \cdot P\left(x_{1}, x_{2}, x_{3}\right)\right)\right)$. This means that from a relation $J\left(P\left(x_{1}, x_{2}, x_{3}\right)\right)=0$ (like the cubic relation (5.10)) and the relation (5.8) we automatically deduce the relations $J\left(\left(x_{1} x_{2} x_{3}\right)^{k} \cdot P\left(x_{1}, x_{2}, x_{3}\right)\right)=0$ for $k \geq 1$. A similar reasoning using the adjoint operators $a d\left(u_{0, l}\right)$ with $l<0$ yields the same equations with now $k \leq-1$. The lemma follows.

Note finally that we do not claim that the positive part $\mathcal{E}^{+}$of $\mathcal{E}$ is isomorphic to the algebra generated by the Fourier coefficients of $\mathbb{T}_{1}(z)$ and $\mathbb{T}_{0}^{+}(z)$ modulo the relevant relations (5.1-5.5). Although this might be the case, we were unable to prove it.

The algebra generated by elements $u_{ \pm 1, d}, \theta_{0, d}$ for $d \in \mathbb{Z}$ subject to the relations (5.15.5) was considered in [5], where it was coined 'quantum continuous $\mathfrak{g l}_{\infty}$ '. There the authors conjectured its link to the stable limit $\mathbf{S} \ddot{\mathbf{H}}_{\infty}$ of spherical DAHAs on the one hand, and to the shuffle algebra $\mathbf{A}_{\tilde{\zeta}(z)}$ on the other. The combination of Theorems 2, 3 with Theorem 4 yields a proof of these conjectures. 


\section{3}

For the sake of completeness, we include here the formulas for the Hopf algebra structure of $\mathcal{E}$. This Hopf algebra structure is inherited from the one which exists on the Hall algebra $\mathbf{H}_{X}$ (see [2]). In terms of the Drinfeld generators, the coproduct takes the following form (see [2, Lemma 4.11]):

$$
\begin{aligned}
\Delta\left(\mathbb{T}_{1}(z)\right) & =\mathbb{T}_{1}(z) \otimes 1+\mathbb{T}_{0}^{+}(z) \otimes \mathbb{T}_{1}(z), \\
\Delta\left(\mathbb{T}_{-1}(z)\right) & =\mathbb{T}_{-1}(z) \otimes 1+\mathbb{T}_{-1}(z) \otimes \mathbb{T}_{0}^{-}(z), \\
\Delta\left(\mathbb{T}_{0}^{ \pm}(z)\right) & =\mathbb{T}_{0}^{ \pm}(z) \otimes \mathbb{T}_{0}^{ \pm}(z) .
\end{aligned}
$$

Note in particular that $\mathcal{E}^{ \pm}$are sub bialgebras of $\mathcal{E}$. The theory of Hall algebras also provides $\mathcal{E}^{ \pm}$with a (topological) antipode. We will not write it here. Finally, note that the $S L(2, \mathbb{Z})$-symmetry is broken if one takes the coproduct into consideration; in other words, $S L(2, \mathbb{Z})$ does not act by Hopf algebra automorphisms (however, the unipotent subgroup $\left(\begin{array}{ll}1 & \mathbb{Z} \\ 0 & 1\end{array}\right)$ does).

\section{Proof of the main theorem}

This section contains the details of the proof of Theorem 4 .

\section{1}

During the course of the proof, we will denote by $\widetilde{\mathcal{E}}$ the algebra generated by the Fourier coefficients of $\mathbb{T}_{-1}(z), \mathbb{T}_{1}(z), \mathbb{T}_{0}^{+}(z)$ and $\mathbb{T}_{0}^{-}(z)$, modulo relations $(5.1-5.4)$ and (5.5). To avoid any confusion with $\mathcal{E}$, we will denote by $\underline{u}_{\epsilon, l}$ for $\epsilon \in\{-1,1\}$ or $\underline{\theta}_{0, l}$ the generators of $\widetilde{\mathcal{E}}$. Since by (5.1) the elements $\underline{\theta}_{0, l}$ commute, we may use (5.7) to define a family of elements $\underline{u}_{0, l}$ for $l \in \mathbb{Z}^{*}$.

The fact that $\mathcal{E}$ is generated by the elements $\left\{u_{ \pm 1, l}, l \in \mathbb{Z}\right\}$ and $\left\{\theta_{(0, l)}, l \in \mathbb{Z}^{*}\right\}$ is a consequence of [2, Corollary 6.1]. That the functional equations (5.1-5.4) hold in $\mathcal{E}$ is also well known-see e.g. [11, Theorem 3.3] or [2, Sect. 6.1]. The relation (5.5) is easy to check directly. Hence there is a natural surjective algebra homomorphism $\phi: \widetilde{\mathcal{E}} \rightarrow \mathcal{E}$ given by $\phi\left(\underline{u}_{\epsilon, l}\right)=u_{\epsilon, l}$ and $\phi\left(\underline{\theta}_{0, l}\right)=\theta_{0, l}$. The content of Theorem 4 is that the map $\phi$ is actually an isomorphism.

\section{2}

We begin with the following Lemma, which will allow us to restrict to positive parts. Let $\widetilde{\mathcal{E}}^{+}$, resp. $\widetilde{\mathcal{E}}^{-}$be the subalgebras of $\widetilde{\mathcal{E}}$ generated by $\left\{\underline{u}_{1, l} \mid l \in \mathbb{Z}\right\}$ and $\left\{\underline{u}_{0, l} \mid l \geq 1\right\}$, resp. by $\left\{\underline{u}_{-1, l} \mid l \in \mathbb{Z}\right\}$ and $\left\{\underline{u}_{0, l} \mid l \leq-1\right\}$. Let also $\widetilde{\mathcal{E}}^{0}$ be the (commutative) subalgebra generated by $\left\{\underline{u}_{0, l} \mid l \in \mathbb{Z}^{*}\right\}$.

Lemma 6.1 The multiplication map

$$
m: \widetilde{\mathcal{E}}^{+} \otimes \widetilde{\mathcal{E}}^{-} \rightarrow \widetilde{\mathcal{E}}
$$

is surjective. 
Proof By (5.4) we have

$$
\left[\underline{u}_{1, l}, \underline{u}_{-1, n}\right] \in \widetilde{\mathcal{E}}^{0} .
$$

Next, by (5.8)

$$
\left[\underline{u}_{0, l}, \underline{u}_{1, n}\right] \in \widetilde{\mathcal{E}}^{+}, \quad\left[\underline{u}_{0, l}, \underline{u}_{-1, n}\right] \in \widetilde{\mathcal{E}}^{-} .
$$

Using (6.2) and (6.3) it is easy to see that one may rewrite any monomial in the variables $\underline{u}_{1, l}, \underline{u}_{0, n}, \underline{u}_{-1, m}$ as a (finite) linear combination of similar monomials in which the variables $\underline{u}_{1, l}, \underline{u}_{0, n}, \underline{u}_{-1, m}$ appear in that order. This proves the Lemma.

Remark Of course, it follows from Theorem 4 that (6.1) is actually an isomorphism.

The map $\phi$ restricts to maps $\phi^{ \pm}: \widetilde{\mathcal{E}}^{ \pm} \rightarrow \mathcal{E}^{ \pm}$, and by Proposition 2.1 and Lemma 6.1 it is enough to show that $\phi^{+}, \phi^{-}$are isomorphisms. Of course, by symmetry it is even enough to deal with $\phi^{+}$.

The algebra $\widetilde{\mathcal{E}}^{+}$is $\mathbb{N}$-graded by the rank since all the defining relations are homogeneous. We denote by $\widetilde{\mathcal{E}}^{+}[r]$ its graded piece of degree $r$, and set $\widetilde{\mathcal{E}}^{+}[\leq r]=$ $\bigoplus_{i=0}^{r} \widetilde{\mathcal{E}}^{+}[i], \widetilde{\mathcal{E}}^{+}[<r]=\bigoplus_{i=0}^{r-1} \widetilde{\mathcal{E}}^{+}[i]$. The spaces $\widetilde{\mathcal{E}}^{-}[s], \widetilde{\mathcal{E}}^{-}[\geq s], \widetilde{\mathcal{E}}^{-}[>s]$ are defined in a similar fashion. We will use the same notations for $\mathcal{E}$ instead of $\widetilde{\mathcal{E}}$. The map $\phi^{+}$restricts to a collection of maps

$$
\phi_{\leq r}^{+}: \widetilde{\mathcal{E}}^{+}[\leq r] \rightarrow \mathcal{E}^{+}[\leq r], \quad \phi_{r}^{+}: \widetilde{\mathcal{E}}^{+}[r] \rightarrow \mathcal{E}^{+}[r]
$$

and similarly for $\phi_{<r}^{+}$.

Our line of proof of Theorem 4 will be to show by induction on $r$ that $\phi_{r}^{+}$(or equivalently $\phi_{\leq r}^{+}$) is an isomorphism. This is clear for $r=0,1$. In this paragraph, we consider the case $r=2$. We will invoke the following result which is a particular case of [2, Lemma 5.6]. Let $\left\{v_{\lambda}\right\}_{\lambda}$ be a $\mathbf{K}$-basis of $\mathcal{E}^{+}[0]=\mathbf{K}\left[u_{0,1}, u_{0,2}, \ldots\right]$.

Lemma 6.2 The collection of elements

$$
\left\{u_{2, l} v_{\lambda} \mid l \in \mathbb{Z}, \lambda\right\} \cup\left\{u_{1, l} u_{1, m} v_{\lambda} \mid l \leq m, \lambda\right\}
$$

forms a $\mathbf{K}$-basis of $\mathcal{E}^{+}[2]$.

Define some elements $\underline{u}_{2, l}$ for $l \in \mathbb{Z}$ through the following formulas:

$$
\underline{u}_{2,2 k+1}=\left[\underline{u}_{1, k+1}, \underline{u}_{1, k}\right], \quad \underline{u}_{2,2 k}=\frac{\alpha_{1}}{\alpha_{2}}\left\{\left[\underline{u}_{1, k+1}, \underline{u}_{1, k-1}\right]-\frac{1}{2} \alpha_{1} \underline{u}_{1, k}^{2}\right\} .
$$


It is clear that $\phi\left(\underline{u}_{2, l}\right)=u_{2, l}$ for $l \in \mathbb{Z}$. Recall that $\phi$ restricts to an isomorphism $\widetilde{\mathcal{E}}^{+}[0] \simeq \mathcal{E}^{+}[0]$ and put $\underline{v}_{\lambda}=\phi^{-1}\left(v_{\lambda}\right)$. We will show that the collection of elements

$$
\left\{\underline{u}_{2, l} \underline{v}_{\lambda} \mid l \in \mathbb{Z}, \lambda\right\} \cup\left\{\underline{u}_{1, l} \underline{u}_{1, m} \underline{v}_{\lambda} \mid l \leq m, \lambda\right\}
$$

linearly generate $\widetilde{\mathcal{E}}^{+}[2]$, from which it will follow that they form a basis, and that $\phi_{\leq 2}$ is indeed an isomorphism. First of all, by (5.8) the elements $\left\{\underline{u}_{1, l} \underline{u}_{1, m} \underline{v}_{\lambda} \mid l, m, \lambda\right\}$ linearly generate $\widetilde{\mathcal{E}}^{+}[2]$. The functional equation (5.3) gives

$$
\begin{aligned}
-\underline{u}_{1, n} \underline{u}_{1, m}+\alpha \underline{u}_{1, n-1} \underline{u}_{1, m+1}-\beta \underline{u}_{1, n-2} \underline{u}_{m+2}+\underline{u}_{1, n-3} \underline{u}_{1, m+3} \\
=-\underline{u}_{1, m} \underline{u}_{1, n}+\beta \underline{u}_{1, m+1} \underline{u}_{1, n-1}-\alpha \underline{u}_{1, m+2} \underline{u}_{n-2}+\underline{u}_{1, m+3} \underline{u}_{1, n-3},
\end{aligned}
$$

where we have set $\alpha=(\sigma \bar{\sigma})^{-1}(\sigma+\bar{\sigma})+\sigma \bar{\sigma}$ and $\beta=\sigma+\bar{\sigma}+(\sigma \bar{\sigma})^{-1}$. We may use (6.6) to try to express a product $\underline{u}_{1, n} \underline{u}_{1, m}$ with $n>m$ as a linear combination of products $\underline{u}_{1, k} \underline{u}_{1, l}$ with $k-l<n-m$. More specifically, (6.6) allows us to express any $\underline{u}_{1, n} \underline{u}_{1, m}$ as a linear combination of elements $\underline{u}_{1, k} \underline{u}_{1, l}$ where $(k, l)$ belongs to either of the sets

$$
\begin{gathered}
\{(n-1, m+1),(n-2, m+2),(n-3, m+3)\}, \\
\{(m+3, n-3),(m+2, n-2),(m+1, n-1),(m, n)\} .
\end{gathered}
$$

From this one deduces that $\sum_{m, n} \mathbf{K}_{1, n} \underline{u}_{1, m}$ is linearly spanned by elements

$$
\begin{aligned}
& \underline{u}_{1, k} \underline{u}_{1, l}, \quad l+k=d, k \leq l+1 \quad(d \text { odd }), \\
& \underline{u}_{1, k} \underline{u}_{1, l}, \quad l+k=d, k \leq l+2 \quad(d \text { even }) \text {. }
\end{aligned}
$$

Comparing (6.4) and (6.7) we see that $\sum_{m, n} \mathbf{K} \underline{u}_{1, n} \underline{u}_{1, m}$ is linearly spanned by $\left\{\underline{u}_{2, l} \mid l \in \mathbb{Z}\right\}$ and $\left\{\underline{u}_{1, l} \underline{u}_{1, m} \mid l \leq m\right\}$ from which it follows in turn that (6.5) generate $\widetilde{\mathcal{E}}^{+}[2]$ as wanted.

\section{4}

For the proof of Theorem 4 we have to introduce several simple combinatorial notions.

Slopes. Let $\mathbf{z}=(r, d)$ be a vector in $\left(\mathbb{Z}^{2}\right)^{+}$. The slope of $\mathbf{z}$ is defined as $\alpha(\mathbf{z})=d / r \in$ $\mathbb{Q} \cup\{\infty\}$. There is a bijection between elements of $\mathbb{Q} \cup\{\infty\}$ and primitive vectors in $\left(\mathbb{Z}^{2}\right)^{+}$.

Farey sequences. Let $F_{0}=\left\{\frac{0}{1}, \frac{1}{0}\right\}, F_{1}=\left\{\frac{0}{1}, \frac{1}{1}, \frac{1}{0}\right\}$, and define $F_{n}$ inductively as follows: between any two consecutive entries $\frac{a}{b}, \frac{c}{d}$ in $F_{n-1}$ insert the element $\frac{a+c}{b+d}$. For instance, we have $F_{2}=\left\{\frac{0}{1}, \frac{1}{2}, \frac{1}{1}, \frac{2}{1}, \frac{1}{0}\right\}, F_{3}=\left\{\frac{0}{1}, \frac{1}{3}, \frac{1}{2}, \frac{2}{3}, \frac{1}{1}, \frac{3}{2}, \frac{2}{1}, \frac{3}{1}, \frac{1}{0}\right\}$. The following facts are classical:

(a) any positive rational number $\frac{p}{q}$ belongs to $F_{n}$ for $n$ large enough, 
(b) for any consecutive entries $\frac{a}{b}, \frac{c}{d}$ in $F_{n}$ we have $a d-b c=-1$,

(c) any pair of positive rational numbers $\left(\frac{a}{b}, \frac{c}{d}\right)$ satisfying $a d-b c=-1$ appears as consecutive entries in some $F_{n}$.

In particular, it follows from (b) that all the fractions appearing in $F_{n}$ are reduced.

Primitive triangles. Let us call a lattice triangle $\Delta_{\mathbf{x}, \mathbf{y}}$ in $\mathbb{Z}^{2}$ with sides $\mathbf{x}, \mathbf{y}, \mathbf{x}+\mathbf{y}$ primitive if it satisfies the condition in relation (2.4), i.e. if $\partial(\mathbf{x})=1$ and if it has no interior lattice point. There are two ways of producing a primitive triangle starting from two consecutive entries $\left(\frac{a}{b}, \frac{c}{d}\right)$ of a Farey sequence $F_{n}$. Namely, let $\mathbf{w}=(b, a), \mathbf{z}=(d, c)$ be the associated primitive vectors in $\left(\mathbb{Z}^{2}\right)^{++}=\left\{(p, q) \in\left(\mathbb{Z}^{2}\right)^{*} \mid p \geq 0, q \geq 0\right\}$. Then

$$
\Delta_{\mathbf{w}, l \mathbf{z}}, \quad \Delta_{\mathbf{z}, l \mathbf{w}} \quad(l>0)
$$

and

$$
\Delta_{\mathbf{z}+l(\mathbf{w}+\mathbf{z}), \mathbf{w}+m(\mathbf{w}+\mathbf{z}), \quad(l, m \geq 0)}
$$

are all primitive triangles.

Lemma 6.3 Any primitive triangle $\Delta_{\mathbf{x}, \mathbf{y}}$ with $\mathbf{x}, \mathbf{y} \in\left(\mathbb{Z}^{2}\right)^{++}$is of the above form (6.8, 6.9) for some consecutive entries of a Farey sequence.

Proof We will use the following result.

Sublemma 6.1 Let $\Delta_{\mathbf{x}, \mathbf{y}}$ be a triangle with no interior lattice points. Then either $\partial(\mathbf{x})=\partial(\mathbf{y})=\partial(\mathbf{x}+\mathbf{y})=2$ or two among $\{\mathbf{x}, \mathbf{y}, \mathbf{x}+\mathbf{y}\}$ are primitive vectors.

Proof It suffices to show that a triangle $\Delta_{\mathbf{u}, \mathbf{v}}$ with $\partial(\mathbf{u})=2, \partial(\mathbf{v})=3$ possesses an interior lattice point. Up to $G L(2, \mathbb{Z})$ action we may assume that $\mathbf{v}=(0,3)$ and that $\mathbf{u}$ belongs to $\left(\mathbb{Z}^{2}\right)^{+}$. Since $\partial(\mathbf{u})=2$ we have $\mathbf{u}=(r, d)$ with $r \geq 2$. Note that $\partial(\mathbf{u}+\mathbf{v}) \leq r$. Pick's formula says that

$$
A=I+\frac{B}{2}-1
$$

where $A$ is the area of $\Delta_{\mathbf{u}, \mathbf{v}}, B=\partial(\mathbf{u})+\partial(\mathbf{v})+\partial(\mathbf{u}+\mathbf{v})$ is the number of boundary points, and $I$ is the number of interior points. In our case, it yields

$$
I=\frac{3}{2} r-\frac{1}{2}(3+2+\partial(\mathbf{u}+\mathbf{v}))+1 \geq \frac{3}{2} r-\frac{1}{2}(3+2+r)+1 \geq r+1-\frac{5}{2} \geq \frac{1}{2} \cdot \square
$$

By the above sublemma, if $\Delta_{\mathbf{x}, \mathbf{y}}$ is a primitive triangle then either $\partial \mathbf{y}=1$ or $\partial(\mathbf{x}+$ $\mathbf{y})=1$. Assume that $\partial(\mathbf{x}+\mathbf{y})=1$. Then from Pick's formula above computing the area of the triangle $\Delta_{\mathbf{x}, \mathbf{y}}$, it follows that $|\operatorname{det}(\mathbf{x}, \mathbf{y})|=\partial(\mathbf{y})$. Hence, if we set $\mathbf{y}_{0}=$ $\mathbf{y} / \partial(\mathbf{y})$ then $\operatorname{det}\left(\mathbf{x}, \mathbf{y}_{0}\right) \in\{-1,1\}$. By the properties of Farey sequence, this means that $\left\{\mathbf{x}, \mathbf{y}_{0}\right\}$ or $\left\{\mathbf{y}_{0}, \mathbf{x}\right\}$ are consecutive entries in some $F_{n}$, and hence that $\Delta_{\mathbf{x}, \mathbf{y}}$ is of the form (6.8). Next assume that $\partial \mathbf{y}=1$ and set $d=\partial(\mathbf{x}+\mathbf{y})$ so that, by Pick's formula, again we have $|\operatorname{det}(\mathbf{x}, \mathbf{y})|=d$. This time, set $(\mathbf{x}+\mathbf{y})_{0}=(\mathbf{x}+\mathbf{y}) / \partial(\mathbf{x}+$ 
$\mathbf{y})$ so that $\left|\operatorname{det}\left(\mathbf{x},(\mathbf{x}+\mathbf{y})_{0}\right)\right|=\left|\operatorname{det}\left(\mathbf{y},(\mathbf{x}+\mathbf{y})_{0}\right)\right|=1$. Up to swapping $\mathbf{x}$ and $\mathbf{y}$ we may assume that $\operatorname{det}\left(\mathbf{x},(\mathbf{x}+\mathbf{y})_{0}\right)=-\operatorname{det}\left(\mathbf{y},(\mathbf{x}+\mathbf{y})_{0}\right)=-1$. Let $l, m$ be the greatest integers satisfying $\mathbf{x}-l(\mathbf{x}+\mathbf{y})_{0} \in\left(\mathbb{Z}^{2}\right)^{++}, \mathbf{y}-m(\mathbf{x}+\mathbf{y})_{0} \in\left(\mathbb{Z}^{2}\right)^{++}$, and put $\mathbf{z}=$ $\mathbf{x}-l(\mathbf{x}+\mathbf{y})_{0}, \mathbf{w}=\mathbf{y}-m(\mathbf{x}+\mathbf{y})_{0}$. We claim that $\operatorname{det}(\mathbf{z}, \mathbf{w})=1$ and that $\mathbf{z}+\mathbf{w}=$ $(\mathbf{x}+\mathbf{y})_{0}$. To see this, first note that $0 \leq r k(\mathbf{z})<r k\left((\mathbf{x}+\mathbf{y})_{0}\right)$. Indeed, it is clear that $0 \leq r k(\mathbf{z}) \leq r k\left((\mathbf{x}+\mathbf{y})_{0}\right)$, and we cannot have $r k(\mathbf{z})=r k\left((\mathbf{x}+\mathbf{y})_{0}\right)$ because otherwise this would imply that $\operatorname{deg}\left((\mathbf{x}+\mathbf{y})_{0}\right)>\operatorname{deg}(\mathbf{z})$ in contradiction with the fact that $\operatorname{det}\left(\mathbf{z},(\mathbf{x}+\mathbf{y})_{0}\right)=\operatorname{det}\left(\mathbf{x},(\mathbf{x}+\mathbf{y})_{0}\right)<0$. For similar reasons, $0<r k(\mathbf{y}-$ $\left.m(\mathbf{x}+\mathbf{y})_{0}\right) \leq r k\left((\mathbf{x}+\mathbf{y})_{0}\right)$. But then comparing ranks and using the equality $\mathbf{x}+\mathbf{y}=$ $\partial(\mathbf{x}+\mathbf{y})(\mathbf{x}+\mathbf{y})_{0}$, we see that $l+m=\partial(\mathbf{x}+\mathbf{y})-1$, which gives $\mathbf{z}+\mathbf{w}=(\mathbf{x}+\mathbf{y})_{0}$. Moreover $\operatorname{det}(\mathbf{z}, \mathbf{w})=\operatorname{det}(\mathbf{x}, \mathbf{y})-(l+m)=1$. This proves our claim. Note that $\mathbf{w}, \mathbf{z}$ are consecutive entries in some Farey sequence since $\operatorname{det}(\mathbf{z}, \mathbf{w})=1$. We are done.

Minimal triangles. Let $\mathbf{z}=(r, d)$ with $r \geq 2$. A primitive triangle $\Delta_{\mathbf{x}, \mathbf{y}}$ in $\mathbb{Z}^{2}$ will be said to be minimal of weight $\mathbf{z}$ if $\mathbf{x}+\mathbf{y}=\mathbf{z}, \operatorname{det}(\mathbf{x}, \mathbf{y})<0$ and if $\partial(\mathbf{x})=\partial(\mathbf{y})=1$. Note that this last condition is automatic if $\partial(\mathbf{z})>1$. It is easy to see using property a) of Farey sequences that there exists minimal triangles of weight $\mathbf{z}$ for any $\mathbf{z}=(r, d)$ with $r \geq 2$.

We will later need the following lemma:

Lemma 6.4 For any $l \geq 1$ there exists a noncommutative polynomial $P_{l}(u, v, w)$, homogeneous of degree $l$ such that the following holds: for any minimal triangle $\Delta_{\mathbf{x}, \mathbf{y}}$ of weight $\mathbf{z}$ we have

$$
P_{l}\left(u_{\mathbf{x}}, u_{\mathbf{z}}, u_{\mathbf{z}+\mathbf{y}}\right)=u_{l \mathbf{z}} .
$$

Proof By $S L(2, \mathbb{Z})$-invariance, we can reduce ourselves to the case $\Delta_{\mathbf{x}, \mathbf{y}} \subset\left(\mathbb{Z}^{2}\right)^{++}$. We are then in the case (6.9) of Lemma 6.3. Without loss of generality, and again by $\operatorname{SL}(2, \mathbb{Z})$-invariance, we may assume that $\mathbf{x}=(p+1, p), \mathbf{y}=(q, q+1)$, with $p, q \in \mathbb{N}$. Observing then that we have a lot of primitive triangles, we check that $u_{(l-2) \mathbf{z}+\mathbf{x}}=\left[u_{\mathbf{z}}, \ldots,\left[u_{\mathbf{z}}, u_{\mathbf{x}}\right] \cdots\right]$ and $\theta_{l \mathbf{z}} / \alpha_{1}=\left[u z+\mathbf{y}, u_{(l-2) \mathbf{z}+\mathbf{x}}\right]$. The existence (and independence on $\Delta_{\mathbf{x}, \mathbf{y}}$ ) of the polynomial $P_{l}$ easily follows.

As examples, we give

$$
P_{1}(u, v, w)=v, \quad P_{2}(u, v, w)=\frac{\alpha_{1}}{\alpha_{2}}\left([u, w]-\frac{\alpha_{1}}{2} v^{2}\right) .
$$

We are now ready to start the proof of Theorem 4. As mentioned above, we argue by induction. Let us fix some $r \in \mathbb{N}$ and let us assume that $\phi_{<r}^{+}$is an isomorphism. By Sect. 5.3, we may in fact assume that $r \geq 3$. For any $\mathbf{x}=(s, l) \in\left(\mathbb{Z}^{2}\right)^{+}$with $0<s<r$ we put $\underline{u}_{\mathbf{x}}=\left(\phi_{<r}^{+}\right)^{-1}\left(u_{\mathbf{x}}\right)$ and $\underline{\theta}_{\mathbf{x}}=\left(\phi_{<r}^{+}\right)^{-1}\left(\theta_{\mathbf{x}}\right)$. By our assumption these elements satisfy the same relations as their counterparts in $\mathcal{E}$ as long as these relations occur in rank strictly smaller than $r$. 
At several points of the proof it will be necessary to consider both elements of $\widetilde{\mathcal{E}}^{+}$and $\widetilde{\mathcal{E}}^{-}$. By symmetry, our assumption implies that the restricted map $\phi_{>-r}^{-}$: $\widetilde{\mathcal{E}}^{-}[>-r] \rightarrow \widetilde{\mathcal{E}}^{-}[>-r]$ is an isomorphism. This allows us to define elements $\underline{u}_{\mathbf{x}}=$ $\left(\phi_{>-r}^{-}\right)^{-1}\left(u_{\mathbf{x}}\right)$ and $\underline{\theta}_{\mathbf{x}}=\left(\phi_{>-r}^{-}\right)^{-1}\left(\theta_{\mathbf{x}}\right)$ for any $\mathbf{x}=(s, l) \in\left(\mathbb{Z}^{2}\right)^{-}$with $-r<s<0$.

Lemma 6.5 For any $s, t>1$ we have

$$
\widetilde{\mathcal{E}}^{+}[<s] \cdot \widetilde{\mathcal{E}}^{-}[>-t]=\widetilde{\mathcal{E}}^{-}[>-t] \cdot \widetilde{\mathcal{E}}^{+}[<s]
$$

and

$$
\mathcal{E}^{+}[<s] \cdot \mathcal{E}^{-}[>-t]=\mathcal{E}^{-}[>-t] \cdot \mathcal{E}^{+}[<s]
$$

Proof Since $\widetilde{\mathcal{E}}^{+}[<s]=\left(\widetilde{\mathcal{E}}^{+}[\leq 1]\right)^{s-1}$ and $\widetilde{\mathcal{E}}^{-}[>-t]=\left(\widetilde{\mathcal{E}}^{-}[\geq-1]\right)^{t-1}$ it suffices to check that $\widetilde{\mathcal{E}}^{+}[\leq 1] \cdot \widetilde{\mathcal{E}}^{-}[\geq-1]=\widetilde{\mathcal{E}}^{-}[\geq-1] \cdot \widetilde{\mathcal{E}}^{+}[\leq 1]$. This is a direct consequence of relations (5.8) and (5.4). The same argument applies to $\mathcal{E}$.

Lemma 6.5 has the following consequence. Let $P\left(u_{\mathbf{x}}\right)$ be a homogeneous polynomial relation in $\mathcal{E}$ involving only generators $u_{\mathbf{x}}$ with $\mathbf{x}=(s, d),|s|<r$, and whose total rank in the elements $u_{\mathbf{x}}$ with $\mathbf{x} \in\left(\mathbb{Z}^{2}\right)^{+}$is strictly less than $r$, resp. whose total rank in the elements $u_{\mathbf{x}}$ with $\mathbf{x} \in\left(\mathbb{Z}^{2}\right)^{-}$is strictly more than $-r$. Then the same relation $P\left(\underline{u}_{\mathbf{x}}\right)$ holds in $\widetilde{\mathcal{E}}$. Indeed, by Lemma 6.5 we have $P\left(\underline{u}_{\mathbf{x}}\right) \in \widetilde{\mathcal{E}}^{+}[<r] \cdot \widetilde{\mathcal{E}}^{-}[>$ $-r]=\widetilde{\mathcal{E}}^{+}[<r] \otimes \widetilde{\mathcal{E}}^{-}[>-r]$ and the restriction of $\phi$ to that space is injective.

6.6

Our method will be to inductively construct elements $\underline{u}_{\mathbf{x}}, \underline{\theta}_{\mathbf{x}}$ for all $\mathbf{x} \in\left(\mathbb{Z}^{2}\right)^{+}$and to verify the relations (2.3-2.4) for all primitive triangles. Our first task will be to define suitable elements in $\widetilde{\mathcal{E}}^{+}$lifting $u_{r, d}, \theta_{r, d} \in \mathcal{E}^{+}$for $d \in \mathbb{Z}$. Our construction of these elements $\underline{u}_{r, d}, \underline{\theta}_{r, d}$ is based on the notion of minimal triangle (see Sect. 6.4). Note that if $\Delta_{\mathbf{x}, \mathbf{y}}$ is a minimal triangle in $\left(\mathbb{Z}^{2}\right)^{+}$of weight $\mathbf{z}$ then $\left[u_{\mathbf{x}}, u_{\mathbf{y}}\right]=\frac{1}{\alpha_{1}} \theta_{\mathbf{z}}$. The idea here is to use this in order to define the desired elements $\underline{u}_{\mathbf{z}}, \underline{\theta}_{\mathbf{z}} \in \widetilde{\mathcal{E}}^{+}[r]$ for $\mathbf{z}=(r, d)$. For this approach to make sense, we need the following result.

Proposition 6.1 Fix $\mathbf{z}=(r, d)$. For any two minimal triangles $\Delta_{\mathbf{x}, \mathbf{y}}$ and $\Delta_{\mathbf{x}^{\prime}, \mathbf{y}^{\prime}}$ of weight $\mathbf{z}$ satisfying $r k(\mathbf{x})>0, r k\left(\mathbf{x}^{\prime}\right)>0$, we have

$$
\left[\underline{u}_{\mathbf{x}}, \underline{u}_{\mathbf{y}}\right]=\left[\underline{u}_{\mathbf{x}^{\prime}}, \underline{u}_{\mathbf{y}^{\prime}}\right] .
$$

Proof Set $l=\partial(\mathbf{z})$ and $\mathbf{w}=\frac{1}{l} \mathbf{z}$. We first assume that $\mathbf{w}=(1, u)$ for some $u \in \mathbb{Z}$. Acting by the unipotent group $\left(\begin{array}{ll}1 & \mathbb{Z} \\ 0 & 1\end{array}\right)$, we may further assume that $u=0$, i.e. that $\mathbf{z}=$ $(l, 0)$. The relevant minimal triangles of weight $\mathbf{z}$ are then the triangles $\Delta_{(i, 1),(l-i, 1)}$ for $i=1, \ldots, l-1$, see Fig. 2 .

If $l=3$ then by (5.5) we have

$$
\left[\underline{u}_{1,0},\left[\underline{u}_{1,1}, \underline{u}_{1,-1}\right]\right]=0=-\left[\underline{u}_{2,1}, \underline{u}_{1,-1}\right]+\left[\underline{u}_{1,1}, \underline{u}_{2,-1}\right]
$$




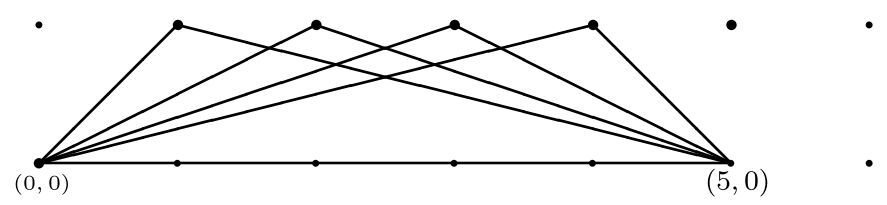

Fig. 2 Minimal triangles of weight $(5,0)$

which is $(6.10)$ in this case. Here we have used the rank two relations

$$
\left[\underline{u}_{1,0}, \underline{u}_{1,1}\right]=-\underline{u}_{2,1}, \quad\left[\underline{u}_{1,0}, \underline{u}_{1,-1}\right]=\underline{u}_{2,-1} .
$$

Suppose now that $l>3$. By the induction hypothesis we have $\left[\underline{u}_{i, 1}, \underline{u}_{l-1-i,-1}\right]=$ $\left[\underline{u}_{i-1,1}, \underline{u}_{l-i,-1}\right]$ for all $i=1, \ldots, l-2$. Applying $\operatorname{ad}\left(\underline{u}_{1,0}\right)$ and using the relations $\left[\underline{u}_{1,0}, \underline{u}_{i, 1}\right]=-\underline{u}_{i+1,1},\left[\underline{u}_{1,0}, \underline{u}_{i+1,-1}\right]=\underline{u}_{i+1,-1}$ for all $i=1, \ldots, l-2$ we get

$$
\left[\underline{u}_{i, 1}, \underline{u}_{l-i,-1}\right]-\left[\underline{u}_{i+1,1}, \underline{u}_{l-i-1,-1}\right]=\left[\underline{u}_{i+1,1}, \underline{u}_{l-i-1,-1}\right]-\left[\underline{u}_{i+2,1}, \underline{u}_{l-i-2,-1}\right]
$$

for $i=1, \ldots, l-3$. We need one more relation in order to be able to deduce that $\left[\underline{u}_{i, 1} \underline{u}_{l-i,-1}\right]=\left[\underline{u}_{j, 1}, \underline{u}_{l-j,-1}\right]$ for all $i, j=1, \ldots, l-1$. If $l=4$ then we get this relation by applying $\operatorname{ad}\left(\underline{u}_{2,0}\right)$ to the equality $\left[\underline{u}_{1,1}, \underline{u}_{1,-1}\right]=\frac{1}{\alpha_{1}} \underline{\theta}_{2,0}$; if $l>4$ then we likewise get this missing relation by applying $\operatorname{ad}\left(\underline{u}_{2,0}\right)$ to $\left[\underline{u}_{i, 1} \underline{u}_{l-2-i,-1}\right]=$ $\left[\underline{u}_{j, 1}, \underline{u}_{l-2-j,-1}\right]$ for all $i, j=1, \ldots, l-3$. This proves Proposition 6.1 when $\mathbf{w}$ is of rank one.

The cases with $r k(\mathbf{w}) \geq 2$ may be dealt with in a similar fashion. If $l=1$ then there is a unique minimal triangle of weight $\mathbf{z}$ and there is nothing to prove. If $l=2$ then there are two minimal triangles $\Delta_{\mathbf{x}, \mathbf{z}-\mathbf{x}}, \Delta_{\mathbf{x}+\mathbf{w}, \mathbf{w}-\mathbf{x}}$; we have $\left[\underline{u}_{\mathbf{x}}, \underline{u}_{\mathbf{w}}\right]=$ $\underline{u}_{\mathbf{x}+\mathbf{w}},\left[\underline{u}_{\mathbf{w}-\mathbf{x}}, \underline{u}_{\mathbf{w}}\right]=-\underline{u}_{\mathbf{Z}-\mathbf{x}}$ and

$$
\left[\underline{u}_{\mathbf{x}}, \underline{u}_{\mathbf{w}-\mathbf{x}}\right]=\underline{u}_{\mathbf{w}} .
$$

The relation $\left[\underline{u}_{\mathbf{x}}, \underline{u}_{\mathbf{z}-\mathbf{x}}\right]=\left[\underline{u}_{\mathbf{x}+\mathbf{w}}, \underline{u}_{\mathbf{w}-\mathbf{x}}\right]$ follows by applying $\operatorname{ad}\left(\underline{u}_{\mathbf{w}}\right)$ to (6.13). Now let us assume that $l \geq 3$. The minimal triangles of weight $\mathbf{z}$ are of the form $\Delta_{\mathbf{x}, l \mathbf{w}-\mathbf{x}}, \Delta_{\mathbf{x}+\mathbf{w},(l-1) \mathbf{w}-\mathbf{x}}, \ldots, \Delta_{\mathbf{x}+(l-1) \mathbf{w}, \mathbf{w}-\mathbf{x}}$, where $\mathbf{x}$ is the point in $\left(\mathbb{Z}^{2}\right)^{+}$of least rank lying on the line $L^{\prime}$ closest to, parallel to, and lying above $L=\mathbb{R} \mathbf{w}$, see Fig. 3 .

By construction, $0<r k(\mathbf{x}) \leq r k(\mathbf{w})$. We claim that $r k(\mathbf{x})<r k(\mathbf{w})$. Indeed if $r k(\mathbf{x})=r k(\mathbf{w})$ then $\mathbf{x}=\mathbf{w}+1$. The parallelogram with sides $\mathbf{w}, \mathbf{x}$ having no interior lattice point and 4 points on its boundary, its area $\operatorname{det}(\mathbf{x}, \mathbf{w})=r k(\mathbf{w})$ is equal to 1. This contradicts our assumption on $\mathbf{w}$ and proves that $0<r k(\mathbf{x})<r k(\mathbf{w})$. Using the induction hypothesis, we have

$$
\left[\underline{u}_{\mathbf{x}}, \underline{u}_{(l-1) \mathbf{w}-\mathbf{x}}\right]=\cdots=\left[\underline{u}_{\mathbf{x}+(l-2) \mathbf{w}}, \underline{u}_{\mathbf{w}-\mathbf{x}}\right]=\frac{1}{\alpha_{1}} \underline{\theta}_{(l-1) \mathbf{w}} .
$$




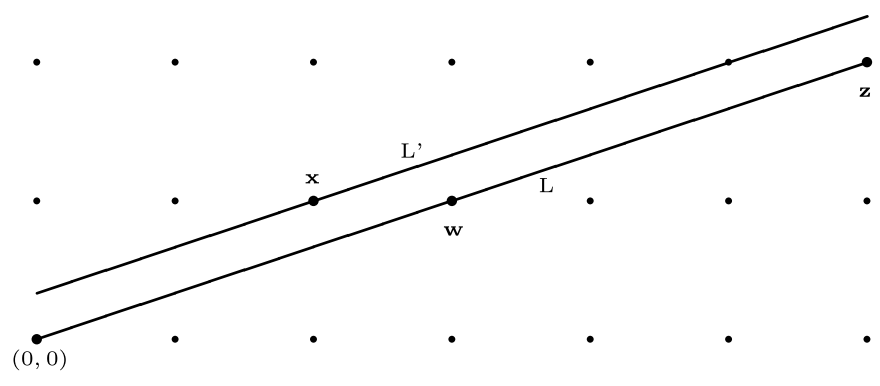

Fig. 3 The line $L^{\prime}$ and the point $x$

Applying $a d\left(\underline{u}_{\mathbf{w}}\right)$ and using some rank $<r$ relations yields

$$
\begin{aligned}
{\left[\underline{u}_{\mathbf{x}+\mathbf{w}}, \underline{u}_{(l-1) \mathbf{w}-\mathbf{x}}\right]-\left[\underline{u}_{\mathbf{x}}, \underline{u}_{l \mathbf{w}-\mathbf{x}}\right] } & =\cdots=\left[\underline{u}_{\mathbf{x}+(l-1) \mathbf{w}}, \underline{u}_{\mathbf{w}-\mathbf{x}}\right]-\left[\underline{u}_{\mathbf{x}+(l-2) \mathbf{w}}, \underline{u}_{2 \mathbf{w}-\mathbf{x}}\right] \\
& =\frac{1}{\alpha_{1}}\left[\underline{u}_{\mathbf{w}}, \underline{\theta}_{(l-1) \mathbf{w}}\right]=\frac{\alpha_{l-1}}{\alpha_{1}}\left[\underline{u}_{\mathbf{w}}, \underline{u}_{(l-1) \mathbf{w}}\right] .
\end{aligned}
$$

Again, we need one more relation. We obtain it by applying $\operatorname{ad}\left(\underline{u}_{(l-1) \mathbf{w}}\right)$ to the equation $\left[\underline{u}_{\mathbf{x}}, \underline{u}_{\mathbf{w}-\mathbf{x}}\right]=\underline{u}_{\mathbf{w}}$, which gives

$$
\left[\underline{u}_{\mathbf{x}+(l-1) \mathbf{w}}, \underline{u}_{\mathbf{w}-\mathbf{x}}\right]-\left[\underline{u}_{\mathbf{x}}, \underline{u}_{l \mathbf{w}-\mathbf{x}}\right]=\left[\underline{u}_{(l-1) \mathbf{w}}, \underline{u}_{\mathbf{w}}\right] .
$$

Combining (6.14) and (6.15) and using the fact that $\alpha_{l-1} / \alpha_{1} \neq-1 /(l-1)$ we obtain $\left[\underline{u}_{\mathbf{w}}, \underline{u}_{(l-1) \mathbf{w}}\right]=0$ as desired. This concludes the proof of Proposition 6.1.

The above proposition allows us to unambiguously define an element $\underline{\theta}_{(r, d)} \in$ $\widetilde{\mathcal{E}}^{+}[r]$ for every $d \in \mathbb{Z}$ by setting

$$
\underline{\theta}_{(r, d)}=\alpha_{1}\left[\underline{u}_{\mathbf{x}}, \underline{u}_{\mathbf{y}}\right]
$$

for any minimal triangle $\Delta_{\mathbf{x}, \mathbf{y}}$ of weight $(r, d)$ with $r k(\mathbf{x})>0$.

Proposition 6.2 If $\mathbf{x}_{1}, \mathbf{x}_{2}$ are colinear vectors in $\left(\mathbb{Z}^{2}\right)^{+}$satisfying $r k\left(\mathbf{x}_{1}\right)+r k\left(\mathbf{x}_{2}\right)=$ $r$ then $\left[\underline{u}_{\mathbf{x}_{1}}, \underline{u}_{\mathbf{x}_{2}}\right]=0$.

Proof If $r=3$ then necessarily $\left\{\mathbf{x}_{1}, \mathbf{x}_{2}\right\}=\{(1, l),(2,2 l)\}$ for some $l \in \mathbb{Z}$, and the fact that $\left[\underline{u}_{(1, l)}, \underline{u}_{(2,2 l)}\right]=0$ is a consequence of relation (5.5). For a general $r$, choose a minimal triangle $\Delta_{\mathbf{z}, \mathbf{x}_{1}-\mathbf{z}}$ of weight $\mathbf{x}_{1}$. We have $\underline{\theta}_{\mathbf{x}_{1}}=\alpha_{1}\left[\underline{u}_{\mathbf{z}}, \underline{u}_{\mathbf{x}_{1}-\mathbf{z}}\right]$. Since the triangle $\Delta_{\mathbf{z}, \mathbf{x}_{1}-\mathbf{z}}$ is minimal there are no lattice points between the line $L$ passing through $\mathbf{x}_{1}$ and $\mathbf{x}_{2}$ and the line $L^{\prime}$ passing through $\mathbf{z}$ and $\mathbf{z}+\mathbf{x}_{2}$. The two triangles $\Delta_{\mathbf{z}, \mathbf{x}_{1}+\mathbf{x}_{2}-\mathbf{z}}$ and $\Delta_{\mathbf{z}+\mathbf{x}_{2}, \mathbf{x}_{1}-\mathbf{z}}$ being both minimal of weight $\mathbf{x}_{1}+\mathbf{x}_{2}$ we have $\left[\underline{u}_{\mathbf{z}}, \underline{u}_{\mathbf{x}_{1}+\mathbf{x}_{2}-\mathbf{z}}\right]=\left[\underline{u}_{\mathbf{z}+\mathbf{x}_{2}}, \underline{u}_{\mathbf{x}_{1}-\mathbf{z}}\right]$ by Proposition 6.1 (Fig. 4). 


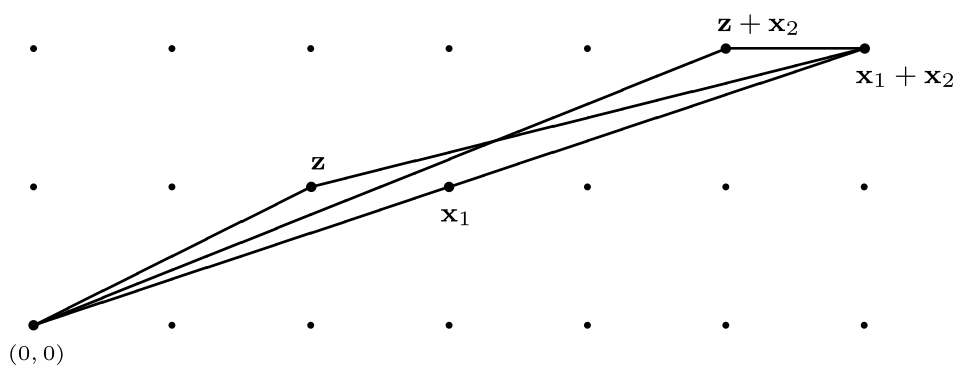

Fig. 4 Two minimal triangles of weight $\mathbf{x}_{1}+\mathbf{x}_{2}$

But then

$$
\begin{aligned}
{\left[\underline{\theta}_{\mathbf{x}_{1}}, \underline{u}_{\mathbf{x}_{2}}\right] } & =\alpha_{1}\left(\left[\left[\underline{u}_{\mathbf{z}}, \underline{u}_{\mathbf{x} 1-\mathbf{z}}\right], \underline{u}_{\mathbf{x}_{2}}\right]\right) \\
& =\alpha_{1}\left(\left[\underline{u}_{\mathbf{z}},\left[\underline{u}_{\mathbf{x}_{1}-\mathbf{z}}, \underline{u}_{\mathbf{x}_{2}}\right]\right]+\left[\left[\underline{u}_{\mathbf{z}}, \underline{u}_{\mathbf{x}_{2}}\right], \underline{u}_{\mathbf{x}_{1}-\mathbf{z}}\right]\right) \\
& =\alpha_{1}\left(-\left[\underline{u}_{\mathbf{z}}, \underline{u}_{\mathbf{x}_{1}+\mathbf{x}_{2}-\mathbf{z}}\right]+\left[\underline{u}_{\mathbf{z}+\mathbf{x}_{2}}, \underline{u}_{\mathbf{x}_{1}-\mathbf{z}}\right]\right)=0 .
\end{aligned}
$$

In the above we have used the fact that there are no lattice points in the interior of the triangles $\Delta_{\mathbf{z}, \mathbf{x}_{2}}, \Delta_{\mathbf{x}_{2}, \mathbf{x}_{1}-\mathbf{z}}$. We have $\underline{\theta}_{\mathbf{x}_{1}}=\alpha_{\partial\left(\mathbf{x}_{1}\right)} \underline{u}_{\mathbf{x}_{1}}+h$ where $h$ is a linear combination of products $\underline{u}_{\mathbf{w}_{1}} \underline{u}_{\mathbf{w}_{2}} \cdots \underline{u}_{\mathbf{w}_{l}}$ with all the $\mathbf{w}_{i}$ s colinear to $\mathbf{x}_{1}$, and $r k\left(\mathbf{w}_{i}\right)<$ $r k\left(\mathbf{x}_{1}\right)$. Hence $\left[\underline{u}_{\mathbf{x}_{2}}, h\right]=0$ and $\left[\underline{u}_{\mathbf{x}_{1}}, \underline{u}_{\mathbf{x}_{2}}\right]=0$ as wanted.

By the above proposition, we may use the relation (5.7) and the construction of $\underline{\theta}_{(r, d)}$ provided by Proposition 6.1 and (6.16) in order to define an element $\underline{u}_{r, d}$.

\section{7}

Now that we have constructed a canonical element $\underline{u}_{(r, d)} \in \widetilde{\mathcal{E}}^{+}[r]$ for every $d \in \mathbb{Z}$, we may proceed with the proof of the induction step. In order to show that $\phi_{\leq r}^{+}$is an isomorphism, we need to check that the relations (2.3), (2.4) hold in $\widetilde{\mathcal{E}}^{+}$. Proposition 6.2 takes care of (2.3), so we will focus on (2.4) for all primitive triangles in $\left(\mathbb{Z}^{2}\right)^{+}$of total rank $r$. The unipotent group $\left(\begin{array}{ll}1 & \mathbb{Z} \\ 0 & 1\end{array}\right)$ acts on $\widetilde{\mathcal{E}}^{+}$and it is easy to see from the definitions that

$$
\left(\begin{array}{cc}
1 & n \\
0 & 1
\end{array}\right) \cdot \underline{u}_{p, q}=\underline{u}_{p, q+n p}
$$

for all $p \leq r$ and $n \in \mathbb{Z}$. Hence it is enough to check that relation (2.4) holds for all primitive triangles in $\left(\mathbb{Z}^{2}\right)^{++}$. Let us fix two vectors $\mathbf{x}, \mathbf{y} \in\left(\mathbb{Z}^{2}\right)^{++}$satisfying $r k(\mathbf{x}+\mathbf{y})=r, \operatorname{det}(\mathbf{x}, \mathbf{y})<0$, and let us assume that either $\Delta_{\mathbf{x}, \mathbf{y}}$ or $\Delta_{\mathbf{y}, \mathbf{x}}$ is primitive. We have to examine the following cases. 


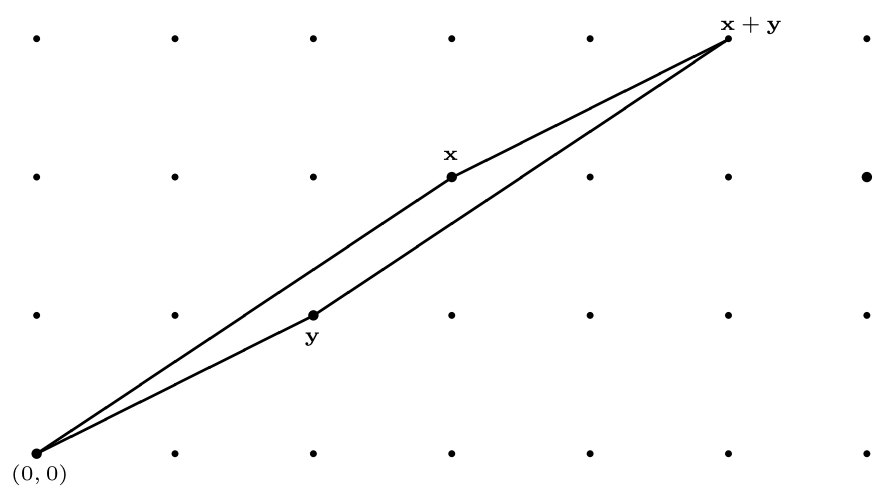

Fig. 5 Case (i): $\mathbf{x}$ and $\mathbf{y}$ are primitive and $r k(\mathbf{x})>0$

Case (i) We have $\partial(\mathbf{x})=\partial(\mathbf{y})=1$. Assume first that $r k(\mathbf{x})>0$. Since $\Delta_{\mathbf{x}, \mathbf{y}}$ is a minimal triangle of weight $\mathbf{x}+\mathbf{y}$, the relation (2.4) in this case is nothing but the definition of $\underline{\theta}_{\mathbf{x}+\mathbf{y}}$ (Fig. 5).

Now let us suppose that $r k(\mathbf{x})=0$, i.e. $\mathbf{x}=(0,1)$. Up to the action of $\left(\begin{array}{ll}1 & n \\ 0 & 1\end{array}\right)$, we may assume that $\mathbf{y}=(r,-1)$. Then we have

$$
\begin{aligned}
{\left[\underline{u}_{0,1}, \underline{u}_{r,-1}\right] } & =\left[\underline{u}_{0,1},\left[\underline{u}_{1,0}, \underline{u}_{r-1,-1}\right]\right] \\
& =\left[\left[\underline{u}_{0,1}, \underline{u}_{1,0}\right], \underline{u}_{r-1,-1}\right]+\left[\underline{u}_{1,0},\left[\underline{u}_{0,1}, \underline{u}_{r-1,-1}\right]\right] \\
& =\left[\underline{u}_{1,1}, \underline{u}_{r-1,-1}\right]+\frac{1}{\alpha_{1}}\left[\underline{u}_{1,0}, \underline{\theta}_{r-1,0}\right] .
\end{aligned}
$$

By Proposition 6.2 we have $\left[\underline{u}_{1,0}, \underline{\theta}_{r-1,0}\right]=0$, from which it follows that $\left[\underline{u}_{0,1}, \underline{u}_{r,-1}\right]$ $=\left[\underline{u}_{1,1}, \underline{u}_{r-1,-1}\right]=\frac{1}{\alpha_{1}} \underline{\theta}_{r, 0}$ as wanted.

Case (i) above exhausts all primitive triangles of the form (6.9). Thus we assume from now on that $\Delta_{\mathbf{x}, \mathbf{y}}$ or $\Delta_{\mathbf{y}, \mathbf{x}}$ is of the form (6.8). In particular, by Lemma 6.3, the slopes $\alpha(\mathbf{y}), \alpha(\mathbf{x})$ are consecutive entries in some Farey sequence, say $F_{n}$.

Case (ii) We have $\partial(\mathbf{y})=\partial(\mathbf{x}+\mathbf{y})=1$ and $\partial(\mathbf{x})>1$. We put $\mathbf{x}_{0}=\mathbf{x} / \partial(\mathbf{x})$ and divide this into subcases as follows:

Case ((ii)a) $\alpha(\mathbf{y})$ appears in an earlier Farey sequence than $\alpha(\mathbf{x})$. Let us set $\mathbf{w}_{0}=$ $\mathbf{x}_{0}-\mathbf{y}$. Then $\alpha(\mathbf{y}), \alpha\left(\mathbf{w}_{0}\right)$ appear as consecutive entries in $F_{n-1}$. In particular, $\mathbf{w}_{0} \in$ $\left(\mathbb{Z}^{2}\right)^{++}$. By our induction hypothesis, we have

$$
\left[\underline{u}_{\partial(\mathbf{x}) \mathbf{w}_{0}}, \underline{u}_{\mathbf{y}}\right]=\underline{u}_{\partial(\mathbf{x}) \mathbf{w}_{0}+\mathbf{y}}
$$

since $r k\left(\mathbf{w}_{0}\right)<r k\left(\mathbf{x}_{0}\right)$ (Fig. 6).

Observe that, in $\mathcal{E}$, relation (6.18) is conjugate under the $S L(2, \mathbb{Z})$ action with the relation $\left[u_{\mathbf{x}}, u_{\mathbf{y}}\right]=u_{\mathbf{x}+\mathbf{y}}$ which we are trying to establish. The idea here is to explicitly realize this conjugation action by suitable adjoint operators, as in the proof of Lemma 5.1. We begin with the following. 


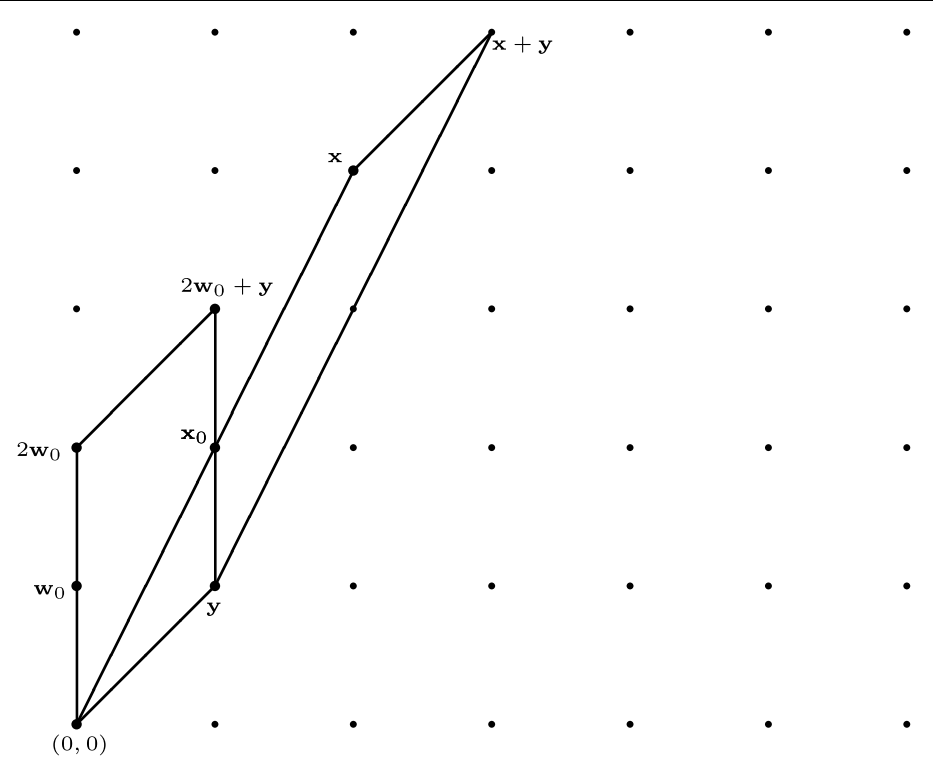

Fig. 6 Case ((ii)a): $\mathbf{y}$ is primitive and $\alpha(\mathbf{x})$ appears after $\alpha(\mathbf{y})$

Lemma 6.6 Let $u_{1}, \ldots, u_{m}, v_{1}, \ldots, v_{s}$ be elements of some algebra A satisfying

$$
\left[v_{i}, u_{l}\right]=v_{i+l} \quad \text { for } i+l \leq s,
$$

$l \leq m$. Then there exists a polynomial $Q_{m}$ in the adjoint operators ad $\left(u_{l}\right), 1 \leq l \leq$ $m$ such that, for any noncommutative homogeneous polynomial $P\left(x_{1}, \ldots, x_{s-m}\right)$ of degree $m$, we have

$$
Q_{m} \cdot P\left(v_{1}, v_{2}, \ldots, v_{s-m}\right)=P\left(v_{2}, v_{3}, \ldots, v_{s-m+1}\right) .
$$

Proof Set $t=s-m$. It is enough to consider the case of a monomial $P\left(v_{1}, \ldots, v_{t}\right)=$ $v_{i_{1}} \cdots v_{i_{m}}$. Denote by $J$ the degree $\leq m$ component of the ring of polynomials $\mathbb{C}\left[y_{1}, \ldots, y_{m}\right]$ and consider the linear map

$$
\begin{aligned}
\kappa: J & \rightarrow A \\
y_{1}^{l_{1}} \cdots y_{m}^{l_{m}} & \mapsto v_{i_{1}+l_{1}} v_{i_{2}+l_{2}} \cdots v_{i_{m}+l_{m}}
\end{aligned}
$$

The assumptions in the lemma imply that for any $F\left(y_{1}, \ldots, y_{m}\right)$ homogeneous of degree at most $m-l$ we have

$$
\operatorname{ad}\left(u_{l}\right) \cdot \kappa\left(F\left(y_{1}, \ldots, y_{m}\right)\right)=-\kappa\left(p_{l} \cdot F\left(y_{1}, \ldots, y_{m}\right)\right)
$$

where $p_{l}=\sum_{i} y_{i}^{l}$ is the power sum symmetric function. For instance

$$
\begin{aligned}
\operatorname{ad}\left(u_{l}\right) \cdot \kappa(1) & =\left[u_{l}, v_{i_{1}} \cdots v_{i_{m}}\right] \\
& =-v_{i_{1}+l} v_{i_{2}} \cdots v_{i_{m}}-v_{i_{1}} v_{i_{2}+l} \cdots v_{i_{m}}-\cdots-v_{i_{1}} \cdots v_{i_{m}+l} .
\end{aligned}
$$


The symmetric function $e_{m}=y_{1} \cdots y_{m}$ belongs to $\mathbb{C}\left[p_{1}, \ldots, p_{m}\right]$ hence there exists a polynomial $Q_{m}$ in the operator $a d\left(u_{1}\right), \ldots, a d\left(u_{m}\right)$ such that $Q_{m} \kappa(1)=\kappa\left(e_{m}\right)$. The lemma is proved.

We apply the above lemma to the following situation: $A=\widetilde{\mathcal{E}}, m=\partial(\mathbf{x}), s=3+m$, $u_{i}=\underline{u}_{i \mathbf{y}}$ for all $i=1, \ldots, \partial(\mathbf{x})$,

$$
v_{1}=\underline{u}_{\mathbf{w}_{0}-\mathbf{y}}, \quad v_{2}=\underline{u}_{\mathbf{w}_{0}}, \quad v_{3}=\underline{u}_{\mathbf{x}_{0}}
$$

and more generally $v_{3+i}=\underline{u}_{\mathbf{x}_{0}+i \mathbf{y}}$. We first need to check that (6.19) holds, that is, that $\left[\underline{u}_{\mathbf{w}_{0}+t \mathbf{y}}, \underline{u}_{l \mathbf{y}}\right]=\underline{u}_{\mathbf{w}_{0}+(l+t) \mathbf{y}}$ whenever $t \geq-1,1 \leq l \leq m$ and $l+t \leq m+1$. For this, assume first that $r k\left(\mathbf{w}_{0}\right)>0$. Then $r k\left(\mathbf{w}_{0}+(m+1) \mathbf{y}\right)<r k\left(m\left(\mathbf{w}_{0}+\mathbf{y}\right)+\mathbf{y}\right)=r$ hence all the above relations are of rank less than $r$ and hold by our induction hypothesis. Note that we could have $\mathbf{w}_{0}-\mathbf{y} \in\left(\mathbb{Z}^{2}\right)^{-}$hence the need to apply Lemma 6.5 (see Sect. 6.5). Next, if $r k\left(\mathbf{w}_{0}\right)=0$, i.e. if $r k\left(\mathbf{x}_{0}\right)=r k(\mathbf{y})$ then $r k\left(\mathbf{w}_{0}+(m+1) \mathbf{y}\right)=r$ so we directly apply the induction hypothesis for all relations except for one, that is, $\left[\underline{u}_{\mathbf{w}_{0}+\mathbf{y}}, \underline{u}_{m \mathbf{y}}\right]=\underline{u}_{\mathbf{w}_{0}+(m+1) \mathbf{y}}$. However, the relation $\left[\underline{u}_{\mathbf{w}_{0}}, \underline{u}_{m \mathbf{y}}\right]=\underline{u}_{\mathbf{w}_{0}+m \mathbf{y}}$ does hold because it is of rank less than $r$, while Proposition 6.2 gives $\left[\underline{u}_{\mathbf{y}}, \underline{u}_{m \mathbf{y}}\right]=0$ so that we indeed have $\left[\underline{u}_{\mathbf{w}_{0}+\mathbf{y}}, \underline{u}_{m \mathbf{y}}\right]=\left[\left[\underline{u}_{\mathbf{w}_{0}}, \underline{u}_{\mathbf{y}}\right], \underline{u}_{m \mathbf{y}}\right]=\left[\left[\underline{u}_{\mathbf{w}_{0}}, \underline{u}_{m \mathbf{y}}\right], \underline{u}_{\mathbf{y}}\right]=\left[\underline{u}_{\mathbf{w}_{0}+m \mathbf{y}}, \underline{u}_{\mathbf{y}}\right]=$ $\underline{u}_{\mathbf{w}_{0}+(m+1) \mathbf{y}}$.

Lemma 6.6 provides us with a polynomial $Q_{m}$ in the adjoint operators $\operatorname{ad}\left(\underline{u}_{\mathbf{y}}\right), \ldots$, $\operatorname{ad}\left(\underline{u}_{m \mathbf{y}}\right)$ such that $Q_{m} \cdot P\left(\underline{u}_{\mathbf{w}_{0}-\mathbf{y}}, \underline{u}_{\mathbf{w}_{0}}, \underline{u}_{\mathbf{x}_{0}}\right)=P\left(\underline{u}_{\mathbf{w}_{0}}, \underline{u}_{\mathbf{x}_{0}}, \underline{u}_{\mathbf{x}_{0}+\mathbf{y}}\right)$ for any homogeneous polynomial $P(u, v, w)$ of degree $m$. We take $P=P_{m}$, defined by Lemma 6.4. Observe that, by the induction hypothesis and rank considerations as above,

$$
\begin{gathered}
P_{m}\left(\underline{u}_{\mathbf{w}_{0}-\mathbf{y}}, \underline{u}_{\mathbf{w}_{0}}, \underline{u}_{\mathbf{x}_{0}}\right)=\underline{u}_{m \mathbf{w}_{0}}, \\
P_{m}\left(\underline{u}_{\mathbf{w}_{0}}, \underline{u}_{\mathbf{x}_{0}}, \underline{u}_{\mathbf{x}_{0}+\mathbf{y}}\right)=\underline{u}_{m \mathbf{x}_{0}}=\underline{u}_{\mathbf{x}} .
\end{gathered}
$$

We therefore have

$$
\underline{u}_{\mathbf{x}}=P_{m}\left(\underline{u}_{\mathbf{w}_{0}}, \underline{u}_{\mathbf{x}_{0}}, \underline{u}_{\mathbf{x}_{0}+\mathbf{y}}\right)=Q_{m} \cdot P_{m}\left(\underline{u}_{\mathbf{w}_{0}-\mathbf{y}}, \underline{u}_{\mathbf{w}_{0}}, \underline{u}_{\mathbf{x}_{0}}\right)=Q_{m} \cdot \underline{u}_{m \mathbf{w}_{0}}
$$

and hence

$$
\left[\underline{u}_{\mathbf{x}}, \underline{u}_{\mathbf{y}}\right]=\left[Q_{m} \cdot \underline{u}_{m \mathbf{w}_{0}}, \underline{u}_{\mathbf{y}}\right]=Q_{m} \cdot\left(\left[\underline{u}_{m \mathbf{w}_{0}}, \underline{u}_{\mathbf{y}}\right]\right)=Q_{m} \cdot \underline{u}_{m \mathbf{w}_{0}+\mathbf{y}}
$$

(recall that, by Proposition 6.2, $\left[\underline{u}_{l \mathbf{y}}, \underline{u}_{\mathbf{y}}\right]=0$ for any $l \leq m$ since $r k((m+1) \mathbf{y}) \leq$ $\left.r k\left(m \mathbf{x}_{0}+\mathbf{y}\right)=r\right)$. Now, $\underline{u}_{m \mathbf{w}_{0}+\mathbf{y}}=\operatorname{ad}\left(\underline{u}_{\mathbf{w}_{0}}\right)^{m}\left(\underline{u}_{\mathbf{y}}\right)$, which we may view as a polynomial in $\underline{u}_{\mathbf{w}_{0}}$ with coefficients in $\mathbb{C}\left[\underline{u}_{\mathbf{y}}\right]$. Since $Q_{m}$ is a polynomial in the adjoint operators $\operatorname{ad}\left(\underline{u}_{\mathbf{y}}\right), \ldots, \operatorname{ad}\left(\underline{u}_{m \mathbf{y}}\right)$ and since $\operatorname{ad}\left(\underline{u}_{l \mathbf{y}}\right) \underline{u}_{\mathbf{y}}=0$ for $l \leq m$, we can apply Lemma 6.6 again, to obtain

$$
Q_{m} \cdot\left(\underline{u}_{m \mathbf{w}_{0}+\mathbf{y}}\right)=Q_{m} \cdot\left(\operatorname{ad}\left(\underline{u}_{\mathbf{w}_{0}}^{m}\right)\left(\underline{u}_{\mathbf{y}}\right)\right)=\operatorname{ad}\left(\underline{u}_{\mathbf{x}_{0}}\right)^{m}\left(\underline{u}_{\mathbf{y}}\right)=\underline{u}_{m \mathbf{x}_{0}+\mathbf{y}}=\underline{u}_{\mathbf{x}+\mathbf{y}}
$$

as wanted. This finishes case ((ii)a).

Case ((ii)b) $\alpha(\mathbf{x})$ appears in an earlier Farey sequence than $\alpha(\mathbf{y})$. If $\mathbf{x}_{0}=(0,1)$ then $\mathbf{y}=(1, n)$ for some $n$ and $r=1$, contrary to our hypothesis. So we may safely assume 


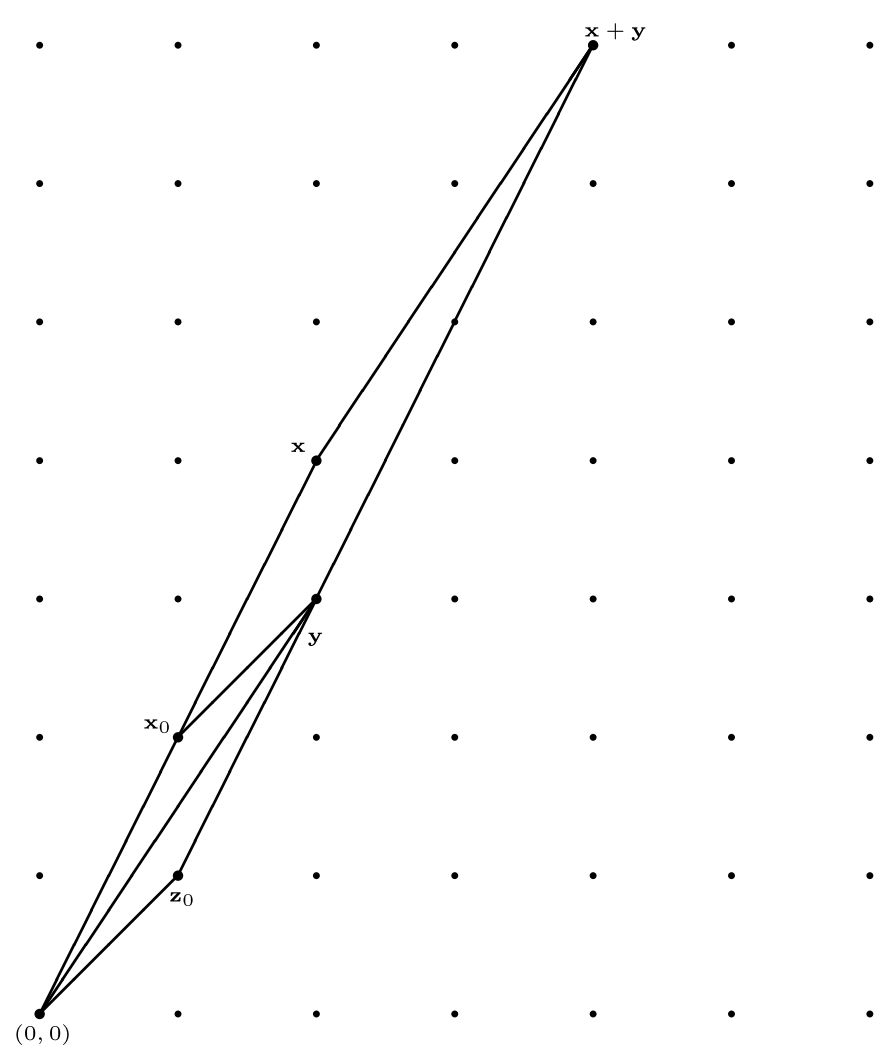

Fig. 7 Case ((ii)b): $\mathbf{y}$ is primitive and $\alpha(\mathbf{x})$ appears before $\alpha(\mathbf{y})$

that $r k\left(\mathbf{x}_{0}\right)>0$. Put $\mathbf{z}_{0}=\mathbf{y}-\mathbf{x}_{0}$. Note that $\alpha\left(\mathbf{z}_{0}\right), \alpha\left(\mathbf{x}_{0}\right)$ are consecutive entries in the Farey sequence $F_{n-1}$ and in particular $\mathbf{z}_{0}$ belongs to $\left(\mathbb{Z}^{2}\right)^{++}$. Moreover, we have $r k\left(\mathbf{x}_{0}\right), r k\left(\mathbf{z}_{0}\right)<r k(\mathbf{y})$. By our induction hypothesis (Fig. 7),

$$
\left[\underline{u}_{\mathbf{x}}, \underline{u}_{\mathbf{z}_{0}}\right]=\underline{u}_{\mathbf{x}+\mathbf{z}_{0}}
$$

since this relation is of rank $r k(\mathbf{x})+r k\left(\mathbf{z}_{0}\right)<r k(\mathbf{x})+r k(\mathbf{y})=r$. Note that $\left[\underline{u}_{\mathbf{x}_{0}}, \underline{u}_{\mathbf{x}}\right]=$ 0 for the same reason since $r k(\mathbf{x})+r k\left(\mathbf{x}_{0}\right)<r k(\mathbf{x})+r k(\mathbf{y})=r$, that $\left[\underline{u}_{\mathbf{x}_{0}}, \underline{u}_{\mathbf{z}_{0}}\right]=\underline{u} \mathbf{y}$, and note that $\left[\underline{u}_{\mathbf{x}_{0}}, \underline{u}_{\mathbf{x}+\mathbf{z}_{0}}\right]=\underline{u}_{\mathbf{x}+\mathbf{y}}$ because $\Delta_{\mathbf{x}_{0}, \mathbf{x}+\mathbf{z}_{0}}$ is a minimal triangle. We now get the desired relation $\left[\underline{u}_{\mathbf{x}}, \underline{u}_{\mathbf{y}}\right]=\underline{u}_{\mathbf{x}+\mathbf{y}}$ by applying $\operatorname{ad}\left(\underline{u}_{\mathbf{x}_{0}}\right)$ to (6.22).

Case ((ii)c) $\alpha\left(\mathbf{x}_{0}\right), \alpha(\mathbf{y})$ appear in $F_{0}$. Then we have $\mathbf{x}_{0}=(0,1), \mathbf{y}=(1,0)$ and therefore $r=1$. This contradicts our assumption on $r$, so this case does not arise.

Case (iii) We have $\partial(\mathbf{x})=\partial(\mathbf{x}+\mathbf{y})=1$ and $\partial(\mathbf{y})>1$.

We put $\mathbf{y}_{0}=\mathbf{y} / \partial(\mathbf{y})$ and further divide this into subcases as follows:

Case ((iii) a) $\alpha(\mathbf{x})$ appears in an earlier Farey sequence than $\alpha(\mathbf{y})$, and $r k(\mathbf{x})>0$ (i.e. $\mathbf{x} \neq(0,1))$. This case is similar to case ((ii)a), with the roles of $\mathbf{x}$ and $\mathbf{y}$ interchanged. We provide the details for the reader's peace of mind. Let us set $\mathbf{z}_{0}=\mathbf{y}_{0}-\mathbf{x}$, so that 


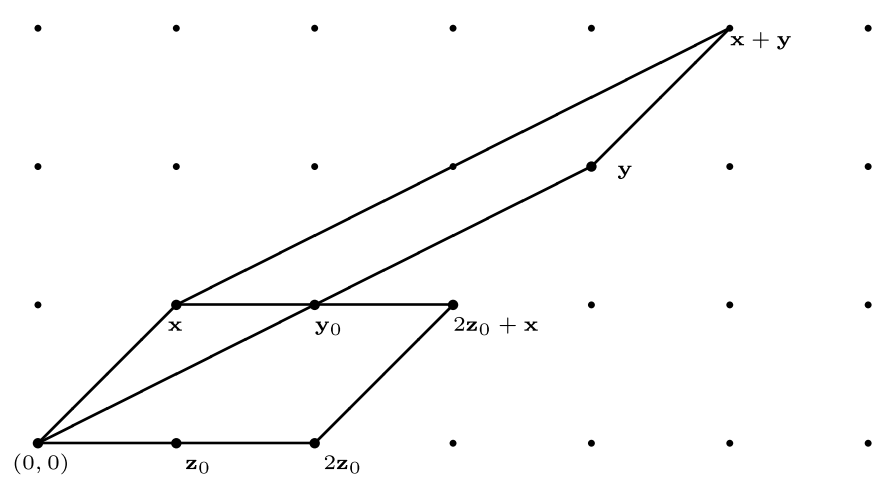

Fig. 8 Case ((iii)a): $\mathbf{x}$ is primitive and $\alpha(\mathbf{y})$ appears after $\alpha(\mathbf{x})$

$\alpha\left(\mathbf{z}_{0}\right), \alpha(\mathbf{x})$ are consecutive entries in $F_{n-1}$. Note that $r k\left(\mathbf{y}_{0}\right)>r k\left(\mathbf{z}_{0}\right)>0$. By our induction hypothesis (Fig. 8),

$$
\left[\underline{u}_{\mathbf{x}}, \underline{u}_{\partial(\mathbf{y}) \mathbf{z}_{0}}\right]=\underline{u}_{\mathbf{x}+\partial(\mathbf{y}) \mathbf{z}_{0}} .
$$

We apply Lemma 6.6 to $A=\widetilde{\mathcal{E}}, n=\partial(\mathbf{y}), s=n+3, u_{i}=\underline{u}_{i \mathbf{x}}$ for $i=1, \ldots, \partial(\mathbf{y})$,

$$
v_{1}=\underline{u}_{\mathbf{z}_{0}-\mathbf{x}}, \quad v_{2}=\underline{u}_{\mathbf{z}_{0}}, \quad v_{3}=\underline{u}_{\mathbf{y}_{0}}
$$

and more generally $v_{3+i}=\underline{u}_{\mathbf{y}_{0}+i \mathbf{x}}$. The requested relations $\left[\underline{u}_{l \mathbf{x}}, \underline{u}_{\mathbf{z}_{0}+d \mathbf{x}}\right]=\underline{u}_{\mathbf{z}_{0}+(l+d) \mathbf{x}}$ for $1 \leq l \leq n, d \geq-1$ and $l+d \leq n+1$ all hold since they are of rank at most $r k\left(\mathbf{z}_{0}+(n+1) \mathbf{x}\right)<r k\left(n\left(\mathbf{z}_{0}+\mathbf{x}\right)+\mathbf{x}\right)=r$. Thus there exists a polynomial $Q_{n}$ in the adjoint operators $\operatorname{ad}\left(\underline{u}_{\mathbf{x}}\right), \ldots, \operatorname{ad}\left(\underline{u}_{n \mathbf{x}}\right)$ such that $Q_{n} \cdot P\left(\underline{u}_{\mathbf{z}_{0}-\mathbf{x}}, \underline{u}_{\mathbf{z}_{0}}, \underline{u}_{\mathbf{y}_{0}}\right)=$ $P\left(\underline{u}_{\mathbf{z}_{0}}, \underline{u}_{\mathbf{y}_{0}}, \underline{u}_{\mathbf{y}_{0}+\mathbf{x}}\right)$ for any homogeneous polynomial $P(u, v, w)$ of degree $n$. We again take $P=P_{n}$, defined by Lemma 6.4. As before, the induction hypothesis implies that

$$
\begin{gathered}
P_{n}\left(\underline{u}_{\mathbf{z}_{0}-\mathbf{x}}, \underline{u}_{\mathbf{z}_{0}}, \underline{u}_{\mathbf{y}_{0}}\right)=\underline{u}_{n \mathbf{z}_{0}}, \\
P_{n}\left(\underline{u}_{\mathbf{z}_{0}}, \underline{u}_{\mathbf{y}_{0}}, \underline{u}_{\mathbf{y}_{0}+\mathbf{x}}\right)=\underline{u}_{n \mathbf{y}_{0}}=\underline{u}_{\mathbf{y}} .
\end{gathered}
$$

We therefore have

$$
\underline{u}_{\mathbf{y}}=P_{n}\left(\underline{u}_{\mathbf{z}_{0}}, \underline{u}_{\mathbf{y}_{0}}, \underline{u}_{\mathbf{y}_{0}+\mathbf{x}}\right)=Q_{n} \cdot P_{n}\left(\underline{u}_{\mathbf{z}_{0}-\mathbf{x}}, \underline{u}_{\mathbf{z}_{0}}, \underline{u}_{\mathbf{y}_{0}}\right)=Q_{n} \cdot \underline{u}_{n \mathbf{z}_{0}}
$$

and hence

$$
\left[\underline{u}_{\mathbf{x}}, \underline{u}_{\mathbf{y}}\right]=\left[\underline{u}_{\mathbf{x}}, Q_{n} \cdot \underline{u}_{n \mathbf{z}_{0}}\right]=Q_{n} \cdot\left(\left[\underline{u}_{\mathbf{x}}, \underline{u}_{n \mathbf{z}_{0}}\right]\right)=Q_{n} \cdot \underline{u}_{n \mathbf{z}_{0}+\mathbf{x}}
$$

(recall that $\left[\underline{u}_{l \mathbf{x}}, \underline{u}_{\mathbf{x}}\right]=0$ for any $l \leq n$ since $\left.r k((n+1) \mathbf{x})<r k\left(n \mathbf{y}_{0}+\mathbf{x}\right)=r\right)$. Now, $\underline{u}_{n \mathbf{z}_{0}+\mathbf{x}}=(-1)^{n} \operatorname{ad}\left(\underline{u}_{\mathbf{z}_{0}}\right)^{n}\left(\underline{u}_{\mathbf{x}}\right)$ so, applying Lemma 6.6 again we have

$$
Q_{n} \cdot\left(\underline{u}_{n \mathbf{z}_{0}+\mathbf{x}}\right)=(-1)^{n} Q_{n} \cdot\left(\operatorname{ad}\left(\underline{u}_{\mathbf{z}_{0}}^{n}\right)\left(\underline{u}_{\mathbf{x}}\right)\right)=(-1)^{n} \operatorname{ad}\left(\underline{u}_{\mathbf{y}_{0}}\right)^{n}\left(\underline{u}_{\mathbf{x}}\right)=\underline{u}_{n \mathbf{y}_{0}+\mathbf{x}}=\underline{u}_{\mathbf{x}+\mathbf{y}}
$$

as wanted. This finishes case ((iii)a). 
Case ((iii)b) $\alpha(\mathbf{y})$ appears in an earlier Farey sequence than $\alpha(\mathbf{x})$. This case is similar to Case ((ii)b). Put $\mathbf{w}_{0}=\mathbf{x}-\mathbf{y}_{0}$. The pair $\left\{\alpha\left(\mathbf{y}_{0}\right), \alpha\left(\mathbf{w}_{0}\right)\right\}$ appears as consecutive entries in $F_{n-1}$, and in particular $\mathbf{w}_{0} \in\left(\mathbb{Z}^{2}\right)^{++}$. Since $r k\left(\mathbf{y}_{0}\right)>0$ we have $r k\left(\mathbf{w}_{0}\right)<$ $r k(\mathbf{x})$. Hence by the induction hypothesis (Fig. 9)

$$
\left[\underline{u}_{\mathbf{w}_{0}}, \underline{u}_{\mathbf{y}}\right]=\underline{u}_{\mathbf{w}_{0}+\mathbf{y}} .
$$

Now observe that $\left[\underline{u}_{\mathbf{y}}, \underline{u}_{\mathbf{y}_{0}}\right]=0$ since $r k(\mathbf{y})+r k\left(\mathbf{y}_{0}\right) \leq r k(\mathbf{y})+r k(\mathbf{x})=r$ (see Proposition 6.2), and note that $\left[\underline{u}_{\mathbf{w}_{0}+\mathbf{y}}, \underline{u}_{\mathbf{y}_{0}}\right]=\underline{u}_{\mathbf{x}+\mathbf{y}}$ since $\Delta_{\mathbf{w}_{0}+\mathbf{y}, \mathbf{y}_{0}}$ is a minimal triangle. The desired relation $\left[\underline{u}_{\mathbf{x}}, \underline{u}_{\mathbf{y}}\right]=\underline{u}_{\mathbf{x}+\mathbf{y}}$ is now obtained by applying $\operatorname{ad}\left(\underline{u}_{\mathbf{y}_{0}}\right)$ to (6.23).

Case $(($ iii $) \mathbf{c}) \mathbf{x}=(0,1), \mathbf{y}_{0}=(1, n)$. Up to the $S L(2, \mathbb{Z})$-action, we can take $n=0$. Hence $\mathbf{y}=(r, 0)$ (Fig. 10).

We begin with the following result.

Claim We have $\left[\underline{u}_{1,0}, \underline{u}_{r, 0}\right]=0$.

Proof of Claim It goes along the lines of the proof of Lemma 6.1. By construction we have $\underline{\theta}_{r, 0}=\alpha_{1}\left[\underline{u}_{s, 1}, \underline{u}_{r-s,-1}\right]$ for all $s=1, \ldots, r-1$. Applying $\operatorname{ad}\left(\underline{u}_{1,0}\right)$ to this equation we get

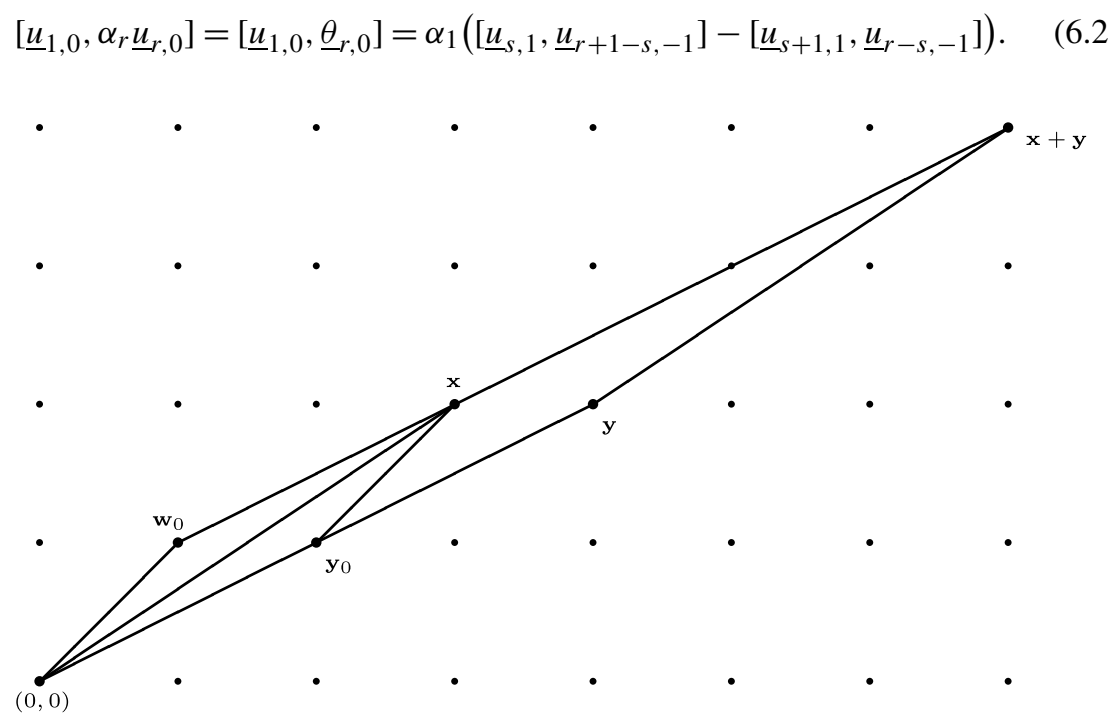

Fig. 9 Case ((ii)b): $\mathbf{x}$ is primitive and $\alpha(\mathbf{x})$ appears after $\alpha(\mathbf{y})$

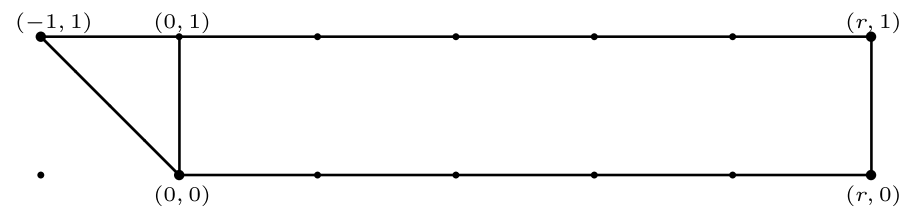

Fig. 10 Case $(($ iii $) \mathrm{c}): \mathbf{x}=(0,1)$ and $\mathbf{y}=(r, 0)$ 
Assume that $r+1=2 k$ is even. Then, by Proposition 6.2 we have $\left[\underline{u}_{k, 0}, \underline{\theta}_{k, 0}\right]=$ $\left[\underline{u}_{k, 0}, \underline{u}_{k, 0}\right]=0$ and by a reasoning similar to the one above,

$$
0=-\left[\underline{u}_{k+1,1}, \underline{u}_{k-1,-1}\right]+\left[\underline{u}_{1,1}, \underline{u}_{2 k-1,-1}\right]
$$

(we use case ((ii)a) to deduce that $\left[\underline{u}_{k, 0}, \underline{u}_{k-1,-1}\right]=\underline{u}_{2 k-1,-1}$ ). Combining (6.24) and (6.25) we obtain

$$
0=k \alpha_{r} / \alpha_{1}\left(\left[\underline{u}_{1,0}, \underline{u}_{r, 0}\right]\right)
$$

which proves our claim in this case.

Now assume that $r=2 k$ is even. Hence $k \geq 2$. We compute on the one hand

$$
\left[\underline{u}_{k, 0}, \underline{u}_{k+1,0}\right]=\frac{1}{\alpha_{k+1}}\left[\underline{u}_{k, 0}, \underline{\theta}_{k+1,0}\right]=\frac{\alpha_{1}}{\alpha_{k+1}}\left(-\left[\underline{u}_{k+1,1}, \underline{u}_{k,-1}\right]+\left[\underline{u}_{1,1}, \underline{u}_{2 k,-1}\right]\right)
$$

(we use Case ((ii)b) to deduce that $\left.\left[\underline{u}_{k, 0}, \underline{u}_{k,-1}\right]=\underline{u}_{2 k,-1}\right)$ and on the other hand

$$
\left[\underline{u}_{k, 0}, \underline{u}_{k+1,0}\right]=\frac{1}{\alpha_{k}}\left[\underline{\theta}_{k, 0}, \underline{u}_{k+1,0}\right]=\frac{\alpha_{1}}{\alpha_{k+1}}\left(\left[\underline{u}_{k+2,1}, \underline{u}_{k-1,-1}\right]-\left[\underline{u}_{1,1}, \underline{u}_{2 k,-1}\right]\right)
$$

(we use case ((ii)b) to deduce that $\left.\left[\underline{u}_{k-1,-1}, \underline{u}_{k+1,0}\right]=-\underline{u}_{2 k,-1}\right)$. Now, using (6.24) we obtain that

$$
\left[\underline{u}_{k, 0}, \underline{u}_{k+1,0}\right]=\frac{k \alpha_{r}}{\alpha_{k+1}}\left[\underline{u}_{1,0}, \underline{u}_{r, 0}\right]
$$

on the one hand while at the same time

$$
\left[\underline{u}_{k, 0}, \underline{u}_{k+1,0}\right]=-\frac{(k+1) \alpha_{r}}{\alpha_{k}}\left[\underline{u}_{1,0}, \underline{u}_{r, 0}\right] .
$$

Since $k / \alpha_{k+1} \neq-(k+1) / \alpha_{k}$ we finally deduce that $\left[\underline{u}_{1,0}, \underline{u}_{r, 0}\right]=0$ as wanted.

Next, we claim that $\left[\underline{u}_{-1,1}, \underline{u}_{r, 0}\right]=\underline{u}_{r-1,1}$. Indeed, we may express $\underline{u}_{r, 0}$ as a polynomial $P=P\left(\underline{u}_{1,0}, \underline{u}_{1, \pm 1}, \ldots\right)$ of degree $r$ in the generators $\underline{u}_{1, l}$ for $l \in \mathbb{Z}$. Then, using relation (5.4) we may express the commutator $\left[\underline{u}_{-1,1}, P\left(\underline{u}_{1,0}, \underline{u}_{1, \pm 1}, \ldots\right)\right]$ as a polynomial in generators $\underline{u}_{0, l}, \underline{u}_{1, \pm l}$ for $l \in \mathbb{Z}$. This polynomial is of degree $r-1$ in the variables $\underline{u}_{1, \pm l}$. Since $\left[u_{-1,1}, u_{r, 0}\right]=u_{r-1,0}$ and since $\phi_{r-1}^{+}$is an isomorphism we deduce that $\left[\underline{u}_{-1,1}, \underline{u}_{r, 0}\right]=\underline{u}_{r-1,1}$ as wanted. Now we apply $\operatorname{ad}\left(\underline{u}_{1,0}\right)$ and use the fact proved above that $\left[\underline{u}_{1,0}, \underline{u}_{r, 0}\right]=0$ to get

$$
\left[\underline{u}_{0,1}, \underline{u}_{r, 0}\right]=\left[\left[\underline{u}_{-1,1}, \underline{u}_{1,0}\right], \underline{u}_{r, 0}\right]=\left[\left[\underline{u}_{-1,1}, \underline{u}_{r, 0}\right], \underline{u}_{1,0}\right]=\left[\underline{u}_{r-1,1}, \underline{u}_{1,0}\right]=\underline{u}_{r, 1}
$$

as desired. This settles case ((iii)c) and with it, finally (!) concludes the proof of Theorem 4.

Acknowledgements I would like to thank A. Tsymbaliuk for useful discussions and for pointing out the paper [5] to me. Special thanks are due to Pierre Baumann for pointing out some flaw in a previous version of this paper. I would also like to acknowledge the referee for his/her help in correcting and improving this paper. 


\section{References}

1. Baumann, P., Kassel, C.: The Hall algebra of the category of coherent sheaves on the projective line. J. Reine Angew. Math. 533, 207-233 (2001)

2. Burban, I., Schiffmann, O.: On the Hall algebra of an elliptic curve, I. Preprint math.AG/0505148 (2005), to appear in Duke Math. J.

3. Drinfeld, V.: A new realization of Yangians and of quantum affine algebras. Sov. Math. Dokl. 36(2), 212-216 (1988)

4. Feigin, B., Odesskii, A.: Vector bundles on an elliptic curve and Sklyanin algebras. In: Topics in Quantum Groups and Finite-Type Invariants. Am. Math. Soc. Transl. Ser. 2, vol. 185, pp. 65-84. Am. Math. Soc., Providence (1998)

5. Feigin, B., Feigin, E., Jimbo, M., Miwa, T., Mukhin, E.: Quantum continuous $\mathfrak{g l}_{\infty}$ : semi-infinite construction of representations. Preprint arXiv:1002.3100 (2010)

6. Feigin, B., Feigin, E., Jimbo, M., Miwa, T., Mukhin, E.: Quantum continuous $g l_{\infty}$ : tensor products of Fock modules and $W_{n}$ characters. Preprint arXiv:1002.3113 (2010)

7. Feigin, B., Hoshino, A., Shibahara, J., Shiraishi, J., Yanagida, S.: Kernel function and quantum algebras. Preprint arXiv:1002.2485 (2010)

8. Feigin, B., Hashizume, K., Hoshino, A., Shiraishi, J., Yanagida, S.: A commutative algebra on degenerate $C P^{1}$ and Macdonald polynomials. J. Math. Phys. 50, 095215 (2009)

9. Feigin, B., Tsymbaliuk, A.: Heisenberg action in the equivariant K-theory of Hilbert schemes via Shuffle Algebra. Preprint arXiv:0904.1679 (2009)

10. Fratila, D.: Cusp eigenforms for elliptic curves and Hall algebras. Preprint (2011)

11. Kapranov, M.: Eisenstein series and quantum affine algebras. J. Math. Sci. 84, 1311-1360 (1997)

12. Ringel, C.: Hall algebras and quantum groups. Invent. Math. 101(3), 583-591 (1990)

13. Schiffmann, O.: Lectures on Hall algebras. Preprint arXiv:math/0611617 (2006), to appear in Sém. \& Cong. (SMF)

14. Schiffmann, O., Vasserot, E.: The elliptic Hall algebra, Cherednik Hecke algebras and Macdonald polynomials. Compos. Math. 147, 188-234 (2011)

15. Schiffmann, O., Vasserot, E.: The elliptic Hall algebra and the equivariant K-theory of the Hilbert scheme of $\mathbb{A}^{2}$. Preprint arXiv:0905.2555 (2009)

16. Schiffmann, O., Vasserot, E.: Hall algebras of curves, commuting varieties and Langlands duality. Preprint arXiv:1009.0678 (2010) 